\title{
JPEG 2000 CODEC Certification Guidance for 1000 ppi Fingerprint Friction Ridge Imagery
}

Shahram Orandi John Libert

John Grantham

Michael Garris

Fred Byers

This publication is available free of charge from: https://doi.org/10.6028/NIST.SP.500-300-upd 


\title{
JPEG 2000 CODEC Certification Guidance for 1000 ppi Fingerprint Friction Ridge Imagery
}

\author{
Shahram Orandi \\ John Libert \\ Michael Garris \\ Fred Byers \\ Information Access Division \\ Information Technology Laboratory
}

John Grantham

Systems Plus, Inc.

Rockville, $M D$

This publication is available free of charge from: https://doi.org/10.6028/NIST.SP.500-300-upd

May 2021

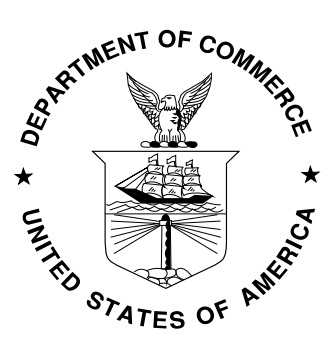

U.S. Department of Commerce Gina M. Raimondo, Secretary

National Institute of Standards and Technology James K. Olthoff, Performing the Non-Exclusive Functions and Duties of the Under Secretary of Commerce for Standards and Technology \& Director, National Institute of Standards and Technology 
Certain commercial entities, equipment, or materials may be identified in this document in order to describe an experimental procedure or concept adequately. Such identification is not intended to imply recommendation or endorsement by the National Institute of Standards and Technology, nor is it intended to imply that the entities, materials, or equipment are necessarily the best available for the purpose.

National Institute of Standards and Technology Special Publication 500-300

Natl. Inst. Stand. Technol. Spec. Publ. 500-300, 36 pages (May 2021) CODEN: NSPUE2

This publication is available free of charge from: https://doi.org/10.6028/NIST.SP.500-300-upd 


\section{VERSION HISTORY}

\begin{tabular}{|l|l|}
\hline Date & Activity \\
\hline $05 / 2015$ & Original document published \\
\hline $03 / 2016$ & $\begin{array}{l}\text { Several changes made to improve accuracy of tabled values and clarity of the conformance test } \\
\text { procedures. }\end{array}$ \\
\hline $08 / 2020$ & $\begin{array}{l}\text { Several updates made to reflect a change in the Reference Fingerprint Image Set, described in section } \\
\text { 3.1, including updates to Table 2, Table 7, Table 8, Table 14, Table 17, Table 19, Table 21, and Table } \\
\text { 22 - Table 25. An update clarifying the application of the Peak Pixel Difference metric was also made } \\
\text { in section 5.2.3. }\end{array}$ \\
\hline & \\
\hline
\end{tabular}




\section{TABLE OF ContentS}

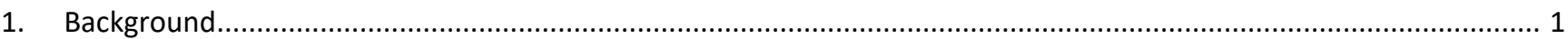

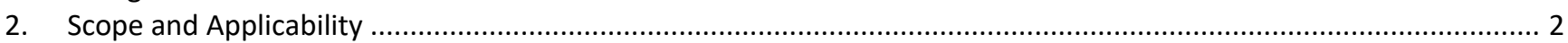

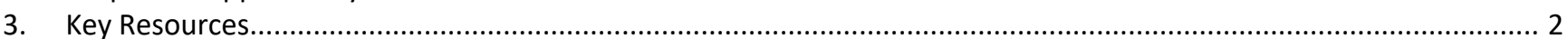

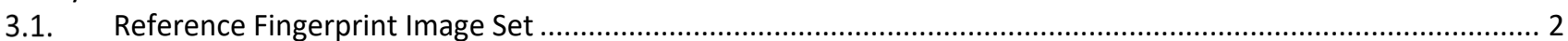

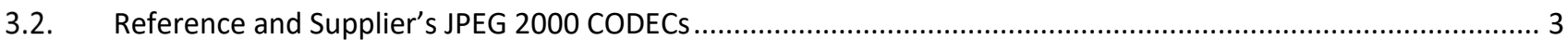

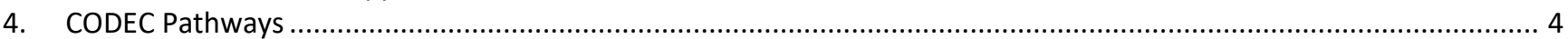

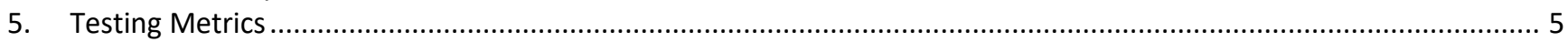

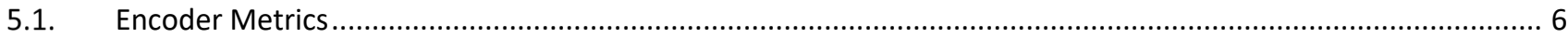

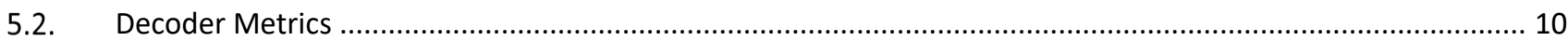

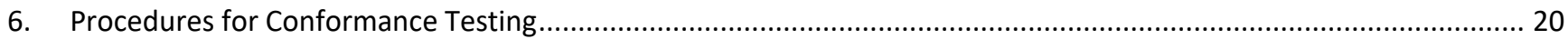

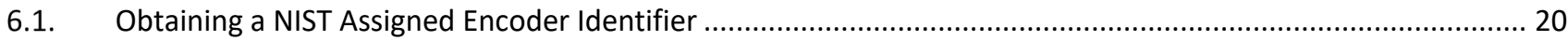

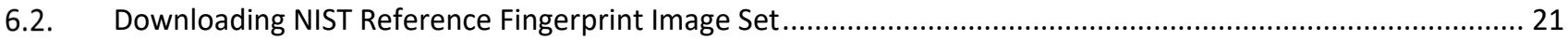

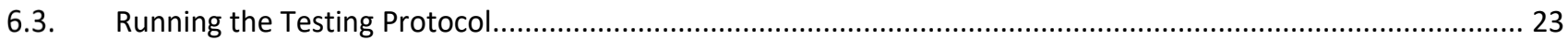

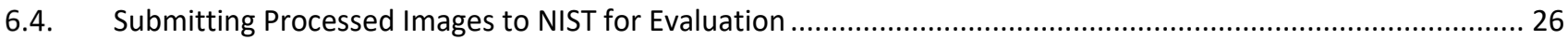

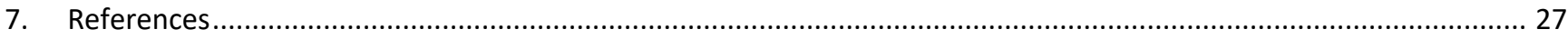

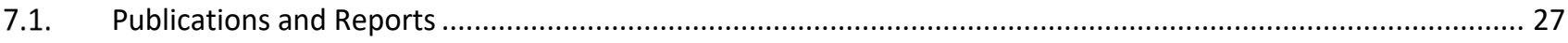

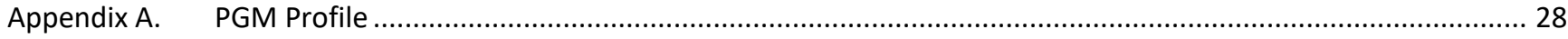




\section{LIST OF TABLES}

Table 1 - Abbreviations

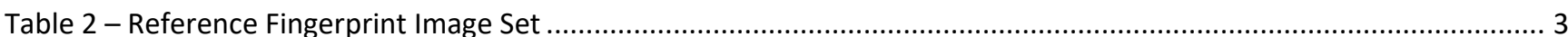

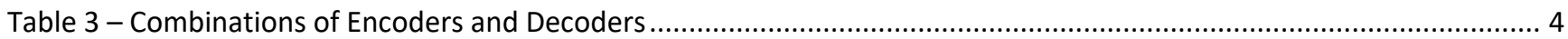

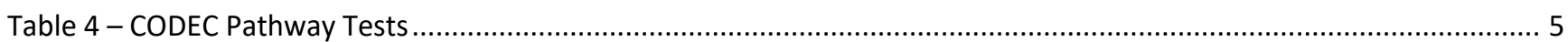

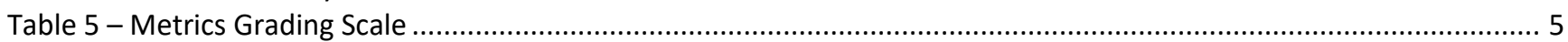

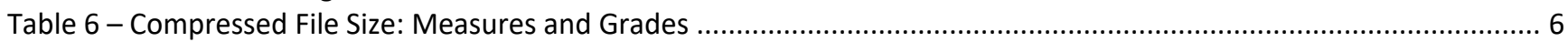

Table 7 - Lossy Compressed File Size: Reference Image Set Values and Thresholds.......................................................... 7

Table 8 - Lossless Compressed File Size: Reference Image Set Values and Thresholds .................................................... 8

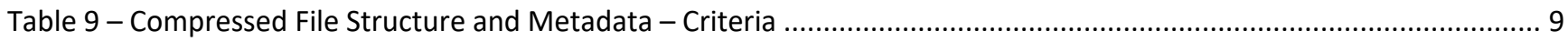

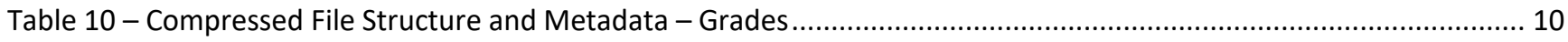

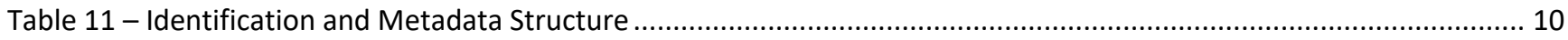

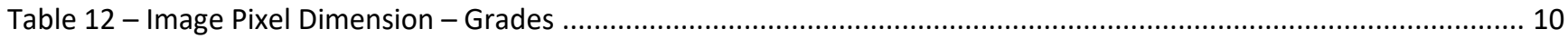

Table 13 - Altered Pixel Count (Lossy): Measures and Grades ....................................................................................... 11

Table 14 - Altered Pixel Count (Lossy): Reference Image Set Values and Thresholds .................................................... 12

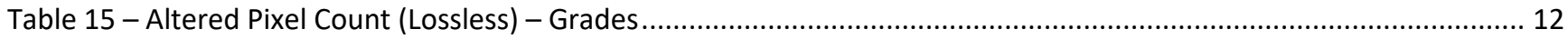

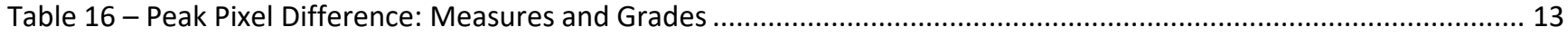

Table 17 - Peak Pixel Difference: Reference Image Set Values and Threshold ............................................................14

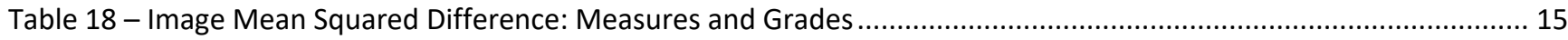

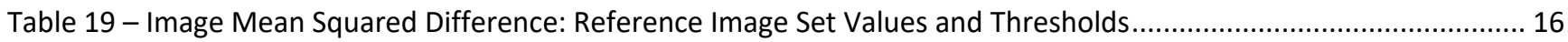

Table 20 - Spectral Image Root Mean Squared Difference: Measures and Grades ........................................................ 18

Table 21 - Spectral Image Root Mean Squared Difference: Reference Image Set Values and Thresholds ......................... 19

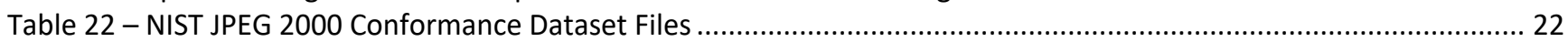

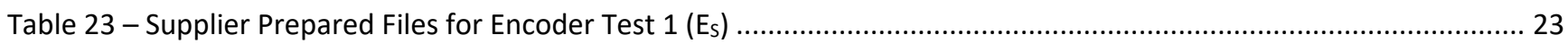

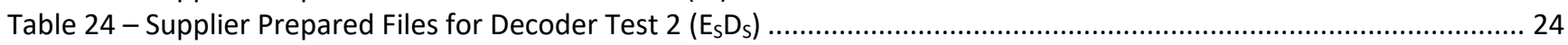

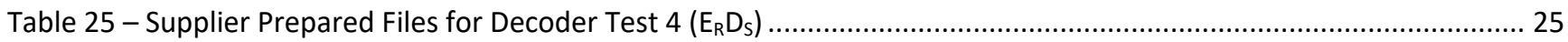

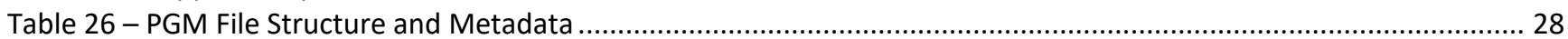

\section{LIST OF FIGURES}

Figure 1 - CODEC Pathways and Input / Output Images.......................................................................................... 4

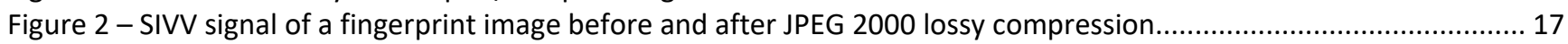

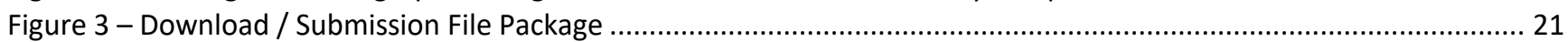




\section{TERMS AND DEFINITIONS}

\section{Table 1 - Abbreviations}

\begin{tabular}{|l|l|}
\hline CJIS & Criminal Justice Information Services Division \\
\hline CODEC & Encoder and Decoder \\
\hline FBI & Federal Bureau of Investigation \\
\hline IAI & International Association for Identification \\
\hline ITL & Information Technology Laboratory \\
\hline JPEG & Joint Photographic Experts Group - ISO/IEC committee developing standards for image compression \\
\hline NBIS & NIST Biometric Image Software \\
\hline NIST & National Institute of Standards and Technology \\
\hline PGM & Portable Graymap (image) Format \\
\hline ppi & Pixels per inch \\
\hline ppmm & Pixels per millimeter \\
\hline SIVV & Spectral Image Validation/Verification Metric \\
\hline WSQ & Wavelet Scalar Quantization - algorithm for compression of fingerprint imagery \\
\hline
\end{tabular}




\section{ABSTRACT}

The document describes the procedure by which applications of JPEG 2000 CODECs will be evaluated with respect to conformance to the NIST guidance for compression of $1000 \mathrm{ppi}^{1}$ friction ridge images as detailed in NIST Special Publication 500-289 [NIST3]. The document describes the attributes of a set of fingerprint images selected for conformance testing and the rationale for selection of these images based on both examiner assessment of image quality over increasing degrees of JPEG 2000 compression and relative fidelity based on computational metrics described SP 500-289 and supporting studies. The document provides background behind the conformance testing, describes the CODEC pathways to be tested and the metrics used to measure conformance, and provides instructions on how to run the protocol and submit results to NIST for evaluation.

\section{KEYWORDS}

Fingerprint lossy compression; lossless compression; 1000 ppi fingerprint imagery; JPEG 2000; latent fingerprints

\footnotetext{
${ }^{1} \mathrm{ppi}=$ pixels per inch; 1000 ppi equals 39.4 pixels per millimeter (ppmm). Resolution values for fingerprint imagery are specified in ppi throughout this document. This is based on widely used specification guidelines for such imagery and is accepted as common nomenclature within the industry.
} 


\section{Background}

The criminal justice communities throughout the world exchange fingerprint imagery data primarily in 8-bit gray-scale and at 500 ppi. The Wavelet Scalar Quantization (WSQ) Gray-Scale Fingerprint Image Compression Specification [WSQ] is the de facto standard for the compression of 500 ppi fingerprint imagery. WSQ is a "lossy" compression scheme. Lossy compression algorithms employ data encoding methods which discard (lose) some of the data in the encoding process in order to achieve an aggressive reduction in the size of the data package. Decompressing the resulting compressed data yields content that, while degraded, is similar enough to the original that it remains useful for the intended purpose. WSQ allows users to specify how much compression is to be applied to the fingerprint image at the cost of increasingly greater loss in fingerprint image fidelity.

The WSQ Specification provides lossy compression guidance based on an International Association for Identification (IAI) study [FITZPATRICK] conducted in the early 90's. This study assessed the negative impact of increased data loss (due to higher levels of lossy compression) on forensic fingerprint comparisons, and from the study's findings a policy of WSQ 15:1 ${ }^{2}$ lossy fingerprint compression was adopted. As a result, conformance testing [WSQ1, WSQ2] has been designed to check adherence to the WSQ Specification and thereby ensure fidelity and admissibility in courts of law for fingerprint images that have been processed by a specific implementation of a WSQ encoder and decoder (CODEC).

Lossless compression is an alternative to lossy compression where compression is applied in such a way as to fully preserve the data content of the source image, thus avoiding any data loss. Lossless compression is typically applied, for example, to latent fingerprint images where image fidelity is critical to maximizing human examiner comparison ${ }^{3}$ and submission of evidence. While lossy compression can achieve rates higher than 10:1, lossless compression algorithms are able to achieve compression rates only on the order of 2:1.

Fingerprint technology has continued to evolve and advance since the establishment of the WSQ Specification for $500 \mathrm{ppi}$ images. A new generation of fingerprint capture devices now exist that scan fingerprints at $1000 \mathrm{ppi}$ and greater. The increased image resolution is particularly helpful with forensic comparison of fingerprints; however, this comes at the price of $4 \mathrm{X}$ the number of pixels for $1000 \mathrm{ppi}$. Over this same period of time, JPEG 2000 [JPEG2K] was developed as a standard CODEC to improve on the original JPEG image compression standard's discrete cosine transform-based methodology [JPEG] yielding increases in both data compression and subjective image quality. JPEG 2000 provides additional flexibility in the creation and manipulation of the code-stream and is based on the same family of wavelets as WSQ which is used for fingerprint image compression at $500 \mathrm{ppi}$. Given the improved performance, greater flexibility, and commodity-level availability of JPEG 2000, it was desirable to migrate from WSQ to the JPEG 2000 standard for use with 1000 ppi fingerprint imagery. This raises the question, "What should be the guidance for applying JPEG 2000 compression to 1000 ppi fingerprints?"

In 2013, the National Institute of Standards and Technology (NIST) in partnership with the Federal Bureau of Investigation (FBI) concluded an investigation to develop this guidance. NIST conducted a series of methodical studies based on the framework established by the IAI's approach to WSQ. The NIST investigation resulted in a set of specifications to be used as a normative specification for the compression of 1000 ppi friction ridge imagery. These specifications were published in NIST Special Publication 500-289 [NIST3], and this specification was adopted by the FBI's Advisory Policy Board (APB) in June 2014.

While SP 500-289 provides guidance on the parameters used with JPEG 2000 to compress 1000 ppi fingerprints, it does not address testing for conformance. This document describes a conformance testing methodology to validate JPEG 2000 CODECs to ensure they meet the operational characteristics resulting from the application of parameters defined in NIST

\footnotetext{
$215: 1$ was found to be the average compression ratio using a target bit rate of 0.75 bits per pixel. With WSQ the actual compression ratio may vary with a given target bit rate according to the spatial frequency content of the image.

${ }^{3}$ FBI-sponsored Scientific Working Group for Friction Ridge Analysis, Study and Technology (SWGFAST) standards since August 2001 (http://www.swgfast.org/Documents.html) have mandated lossless image compression for latent print examination casework, but exempted AFIS-related fingerprint and palm-print images from lossless compression requirements. As of March 2015, the Friction Ridge Subcommittee of NIST's Organization of Scientific Area Committees is embracing SWGFAST's existing digital imaging standards and guidelines, but may modify them at a future date.
} 
SP 500-289 as applied to 1000 ppi friction ridge imagery. This includes compression in both lossy 10:1 and lossless modes. While SP 500-289 also specifies the preferred method for downsampling 1000 ppi friction ridge imagery to 500 ppi, the conformance testing of downsampling is to be described in NIST Special Publication 500-306.

Section 3 describes the key resources supporting this effort; Section 4 presents a framework of the CODEC pathways that are to be tested; Section 5 defines the testing metrics used to evaluate image fidelity and conformance; Section 6 describes testing reports and the criteria to receive certification (from the FBI); and Section 7 documents procedures for participating in this testing.

\section{Scope and Applicability}

This document covers conformance of JPEG 2000 CODECs to the specification for compression of 1000 ppi friction ridge images as described in NIST Special Publication 500-289 [NIST3]. It excludes elements of the specification relating to the downsampling of 1000 ppi fingerprint images to 500 ppi with subsequent compression according to the WSQ specification [WSO] for interoperability with legacy fingerprint databases. Conformance to downsampling recommendations will be covered in a separate document to be designated NIST Special Publication 500-306.

\section{Key Resources}

\subsection{Reference Fingerprint Image Set}

A carefully selected set of 1000 ppi friction ridge imagery is needed to test implementations of SP 500-289 - specifically to test the proper application of SP 500-289 to a JPEG 2000 CODEC as indicative by measuring the fidelity of resulting images to the original images. To this end, a subset of 30 fingerprint images (listed in Table 2) were selected from NIST Special Database 300 (SD-300) [NIST6] based on their sensitivity to compression changes as measured by spectral frequency comparison of compressed images with non-compressed original fingerprint impressions acquired at $1000 \mathrm{ppi}$ [LIBERT] and confirmed by human assessors.

In [NIST2] we report that trained fingerprint examiners observed in most cases the increased degradation in image quality over the three compression rations, 7:1, 10:1, and 12:1. The analysis of examiner ratings found 10:1 compression the threshold beyond which image degradation became sufficiently significant as to possibly impact latent matching. In selecting images from SD-300, we did not have benefit of large numbers of trained examiners. However, we were able to extend the examiner results to the new dataset by looking for the maximum distortion of image spectra in comparison to non-compressed exemplars with versions our source images compressed at 7:1 and 12:1. The procedure can be summarized as follows:

1. Select a subset of 600 source images at random from SD-300;

2. Apply the JPEG 2000 encoder to each source image at rates 7:1, 10:1, and 12:1;

3. Apply the NIST Spectral Image Verification Validation (SIVV) metric [LIBERT] to extract power spectrum for each of the compressed images as well as that of the non-compressed source;

4. Compute the Root Mean Squared Difference (RMSD) between the power spectra of each of the compressed images and that of its source;

5. For each of the 600 RMSD values, compute the slope of the line fitting the RMSD values at 7:1, 10:1, and 12:1 and sort the values from highest to lowest slope;

6. Select the 200 source images exhibiting the greatest slope in RMSD from 7:1 to 12:1.

7. To confirm that degradation would be visible to observers, the 7:1,10:1, and 12:1 compressed versions of each of the selected 200 source images were presented, in a randomized order of "triplets", to a group of experienced human assessors using customized software for collecting their assessments

8. The group of assessors ranked each image within a triplet from the highest to lowest quality until the entire set of 200 was completed (for each assessor)

9. A subset of 30 source images was selected for which agreement among all assessors was reached

Images selected by the computational method and verified by human inspection are listed in Table 2 below. 
As will be seen in Section 4, a number of the image fidelity metrics employed in this testing use thresholds based on statistical margins computed between measurements observed at 10:1 and 12:1. This serves to set bounds on a range known to impact relevant image fidelity. Certain tests also identify behavior computed between measurement observations at 7:1 and 10:1, especially in cases where performance of the CODEC exceeds expectations of the test.

Table 2 - Reference Fingerprint Image Set

\begin{tabular}{|c|c|c|c|c|}
\hline $\begin{array}{c}\text { Image } \\
\#\end{array}$ & $\begin{array}{c}\text { Impression } \\
\text { Type }\end{array}$ & $\begin{array}{l}\text { Image } \\
\text { W }\end{array}$ & $\underset{H}{\text { Image }}$ & Image File Name \\
\hline 1 & Flat & 540 & 860 & S0011_1000_08P-SRC \\
\hline 2 & Flat & 630 & 930 & S0187_1000_07P-SRC \\
\hline 3 & Flat & 662 & 890 & S0201_1000_02P-SRC \\
\hline 4 & Flat & 720 & 1850 & S0417_1000_11-SRC \\
\hline 5 & Flat & 470 & 870 & S0464_1000_09P-SRC \\
\hline 6 & Flat & 600 & 900 & S0564_1000_03P-SRC \\
\hline 7 & Flat & 560 & 950 & S0586_1000_10P-SRC \\
\hline 8 & Flat & 748 & 1782 & S0676_1000_12-SRC \\
\hline 9 & Flat & 580 & 580 & S0704_1000_05P-SRC \\
\hline 10 & Flat & 590 & 930 & S0730_1000_04P-SRC \\
\hline 11 & Rolled & 1550 & 1496 & S0051_1000_05-SRC \\
\hline 12 & Rolled & 1536 & 1592 & S0093_1000_01-SRC \\
\hline 13 & Rolled & 1618 & 1606 & S0152_1000_06-SRC \\
\hline 14 & Rolled & 1496 & 1498 & S0176_1000_02-SRC \\
\hline 15 & Rolled & 1536 & 1334 & S0249_1000_08-SRC \\
\hline 16 & Rolled & 1524 & 1484 & S0342_1000_09-SRC \\
\hline 17 & Rolled & 1522 & 1402 & S0397_1000_04-SRC \\
\hline 18 & Rolled & 1470 & 1414 & S0503_1000_07-SRC \\
\hline 19 & Rolled & 1482 & 1414 & S0705_1000_03-SRC \\
\hline 20 & Rolled & 1592 & 1400 & S0724_1000_10-SRC \\
\hline 21 & Slap & 3196 & 1890 & S0139_1000_13-SRC \\
\hline 22 & Slap & 3208 & 1986 & S0286_1000_13-SRC \\
\hline 23 & Slap & 3168 & 1958 & S0304_1000_14-SRC \\
\hline 24 & Slap & 3102 & 2014 & S0392_1000_13-SRC \\
\hline 25 & Slap & 3100 & 2000 & S0465_1000_14-SRC \\
\hline 26 & Slap & 3208 & 1986 & S0466_1000_13-SRC \\
\hline 27 & Slap & 3156 & 1890 & S0641_1000_14-SRC \\
\hline 28 & Slap & 3086 & 1850 & S0795_1000_14-SRC \\
\hline 29 & Slap & 3208 & 1986 & S0817_1000_13-SRC \\
\hline 30 & Slap & 3182 & 1836 & S0850_1000_14-SRC \\
\hline
\end{tabular}

\subsection{Reference and Supplier's JPEG 2000 CODECs}

The goal of this testing is to determine whether the specification in SP 500-289 has been successfully applied to a specific JPEG 2000 CODEC. This not only requires a reference set of fingerprint images, but also a "reference" CODEC to which results from a CODEC under evaluation can be compared. In this document, the organization requesting conformance testing is referred to as the "supplier" and the algorithm they desire to be evaluated is referred to as the "supplier's CODEC." (Note that it is not the supplier's software implementation of a JPEG 2000 CODEC that is shared with NIST, but rather it is the images or compressed files processed by the supplier's CODEC that are submitted.)

NIST used the Open JPEG's [OPENJPEG] implementation version 2.1 to test the guidance in SP 500-289, and the same implementation is used as the reference CODEC in this test suite. The Open JPEG CODEC, with minor modification, has been incorporated into the NIST Biometric Image Software (NBIS) public domain software distribution [NIST1] and is freely available to CODEC suppliers. The reference fingerprint image set has been processed by the NIST reference CODEC and the results are used to compare outputs from the supplier's CODEC under evaluation. 


\section{CODEC Pathways}

By definition a CODEC is comprised of two primary functions: encode, ' $E$ ', followed by decode, ' $D$ '. In this evaluation we are comparing performance between two CODECs: the reference CODEC, ' $R$ ', and the supplier's CODEC, ' $S$ '. Encoders are to be independent of Decoders, and in this testing the interoperability of the two CODECs is evaluated. Table 3 lists the possible native and interoperable combinations of encoders and decoders. All combinations except for the first column (the native reference) is of interest and will be evaluated.

Table 3 - Combinations of Encoders and Decoders

\begin{tabular}{c|c|c|c}
\hline \multicolumn{2}{c}{ NATIVE } & \multicolumn{2}{c}{ INTEROPERABLE } \\
\hline $\mathbf{E}_{\mathbf{R}} \mathbf{D}_{\mathbf{R}}$ & $\mathbf{E}_{\mathbf{S}} \mathbf{D}_{\mathbf{S}}$ & $\mathbf{E}_{\mathbf{R}} \mathbf{D}_{\mathbf{S}}$ & $\mathbf{E}_{\mathbf{S}} \mathbf{D}_{\mathbf{R}}$ \\
\hline Reference Encoder & Supplier's Encoder & Reference Encoder & Supplier's Encoder \\
$\downarrow$ & $\downarrow$ & $\downarrow$ & $\downarrow$ \\
Reference Decoder & Supplier's Decoder & Supplier's Decoder & Reference Decoder
\end{tabular}

Regrouping these combinations for processing efficiencies creates the pathways shown in Figure 1 . Here the inputs and outputs are annotated along with the encoders and decoders. The input to the encoders are the reference fingerprint images, which have never been lossy-compressed and are stored in the Portable Graymap Format (PGM) [PGM] format noted as ' $P$ ', with file extension ". pgm". The reference fingerprint images are referred to as "source" images and are noted as ' $P_{\text {SRC }}$. The output from the encoders (and input to the decoders) are JPEG 2000 files with extension ".jp2" and are noted as ' $J$ ' with a subscript corresponding to its parent encoder. For example, a JPEG 2000 file generated by the supplier's encoder is noted, ' $\mathrm{J}_{\mathrm{ES}_{\mathrm{S}}}$. The decoders also output PGM files that reflect the changes (and any degradation) to the source image introduced by the specific CODEC pathway. For example, a PGM file containing results from a fingerprint image which has been lossy-compressed using the supplier's encoder and then processed by the reference decoder is noted, ' $P_{E_{S} D_{R}}$ '. While the reference input fingerprint images are referred to as "source" images, the output images from the encoders and decoders are referred to as "processed" images.

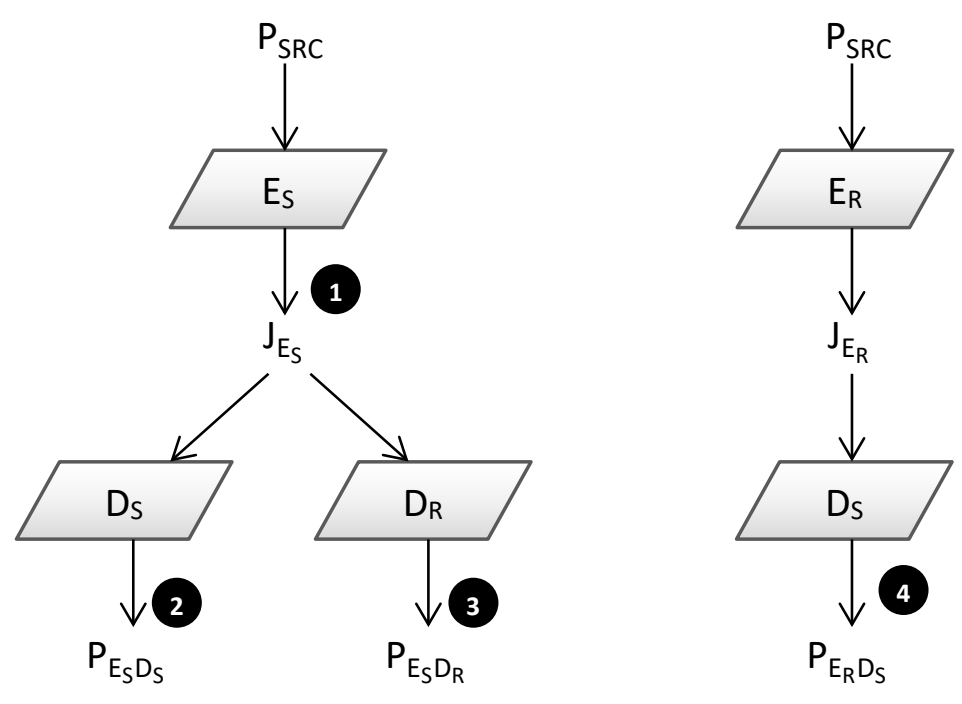

Figure 1 - CODEC Pathways and Input / Output Images

Within these pathways, tests are conducted at four strategic points noted by the circled numbers in the figure above. Table 4 lists the pathway tests along with the images that are compared to assess conformance. The metrics used within each of these tests are documented in Section 5. Note that Test 1 is an encoder-output test, while the other three are decoderoutput tests. Also note that Test 3 will be completed by NIST while Tests 1, 2, and 4 will be completed by Suppliers, with the results being sent to NIST for evaluation. 
Table 4 - CODEC Pathway Tests

\begin{tabular}{c|c|c}
\hline Test & Pathway & Compared \\
\hline 1 & $E_{S}$ & $J_{E_{S}}, J_{E_{R}}$ \\
\hline 2 & $E_{S} D_{S}$ & $P_{E_{S} D_{S}}, P_{S R C}$ \\
3 & $E_{S} D_{R}$ & $P_{E_{S} D_{R}}, P_{S R C}$ \\
\hline 4 & $E_{R} D_{S}$ & $P_{E_{R} D_{S}}, P_{S R C}$ \\
\hline
\end{tabular}

SP 500-289 provides compression specification of 1000 ppi friction ridge imagery for both lossy $10: 1$ and lossless modes. It also sets specification on downsampling from $1000 \mathrm{ppi}$ to $500 \mathrm{ppi}$. The conformance testing for downsampling is addressed in NIST Special Publication 500-306 Certification Guidance for the downsampling of 1000 ppi Fingerprint Friction Ridge Imagery to $500 \mathrm{ppi}$. The testing of both lossy and lossless compression modes follow the same pathways and tests described in Figure 1 and Table 4. The metrics applied to lossy and lossless compression do however differ slightly as described in Section 5.

\section{Testing Metrics}

Unlike WSQ CODECs, the behavior of JPEG 2000 CODECs varies considerably within a band of "acceptable performance." Whereas WSQ compliance could be assessed very tightly using narrowly defined criteria, such performance precision is not possible with JPEG 2000. For example, for the image-based metrics described below, three different JPEG $2000 \mathrm{CODECs}^{4}$ yielded different values when applied to the same test images. As the JPEG 2000 standard itself allows considerable latitude in implementing a CODEC, it would be unfair to define performance criteria tightly to the metric outputs of the NIST (Open JPEG) reference CODEC. Accordingly, for the majority of the image-based metrics used in this testing, performance is assessed by applying a tolerance margin to the measurements made using the reference CODEC. Each image-based metric quantifies a different factor representing change (degradation). Better performing CODECs will generate metrics within a tighter tolerance (or perhaps even exceed the performance of the reference CODEC).

For most of the metrics, conformance is graded at two tolerance thresholds. Those CODECs performing within a primary (tighter) tolerance are granted a grade of "Pass"; those that do not meet this level but are within a secondary (more relaxed) tolerance are granted a grade of "Nominal Pass"; and those that fall outside the secondary tolerance receive a grade of "Fail" for that particular metric. A special designation of "Gold" applies to a Passing supplier's CODEC that demonstrates improved performance over the reference CODEC. Table 5 lists the possible grade categories.

Table 5 - Metrics Grading Scale

\begin{tabular}{|c|c|}
\hline $\begin{array}{c}\text { Special } \\
\text { Designation }\end{array}$ & Grade \\
\hline Gold (Pass) & Desirable performance exceeding that of reference design \\
\hline Pass & Acceptable performance meeting reference design \\
\hline Nominal Pass & Acceptable performance within tolerance of reference design \\
\hline Fail & Unacceptable performance below reference design tolerances \\
\hline
\end{tabular}

The metrics used in this testing apply either to encoders or to decoders. The encoder metrics are file-based while the decoder metrics are image-based. Metrics are applied and grades are assigned for performance on each image or file in the suite of test materials. The final grade for each metric is the lowest single grade achieved among the suite of individual measurements regardless of performance on other images or compressed data files in the test dataset. The philosophy here is that for purposes of assessing conformance, the minimum performance is the best indicator and preferred over average or maximum performance.

${ }^{4}$ In addition to Open JPEG, NIST also explored the use of Jasper [JASPER] and Kakadu ${ }^{\mathrm{TM}}$ [KAKADU]. 


\subsection{Encoder Metrics}

The encoder metrics described below apply to testing both lossy and lossless compression modes.

\subsubsection{Compressed File Size}

This metric $\left(M_{\text {size }}\right)$ evaluates the size of the resulting compressed JPEG 2000 image produced by the supplier's encoder $\left(\mathrm{J}_{\mathrm{Es}}\right)$, determining if the file size (in bytes) is within an acceptable tolerance of that produced by the NIST reference encoder $\left(\mathrm{J}_{\mathrm{E}_{\mathrm{R}}}\right)$. Grades are assessed based on measures set according to Table 6. The thresholds defined in Table 6 are computed across the reference fingerprint image set and listed in Table $7 .^{5}$

Table 6 - Compressed File Size: Measures and Grades

\begin{tabular}{ll}
\hline Value Being Graded & $\mathrm{M}_{\text {size }}\left(\mathrm{J}_{E_{S}}\right)$ \\
Reference Measurement & $\mathrm{M}_{\text {size }}\left(\mathrm{J}_{E_{R}}\right)$ \\
\hline Primary Tolerance & $5 \% \mathrm{M}_{\text {size }}\left(J_{E_{R}}\right)$ \\
Secondary Tolerance & $10 \% \mathrm{M}_{\text {size }}\left(J_{E_{R}}\right)$ \\
\hline Primary Threshold & $\mathrm{M}_{\text {size }}\left(\mathrm{J}_{E_{R}}\right)+$ Primary Tolerance \\
\hline Secondary Threshold & $\mathrm{M}_{\text {size }}\left(\mathrm{J}_{E_{R}}\right)+$ Secondary Tolerance \\
\hline Gold (Pass) & $<=99 \% \mathrm{M}_{\text {size }}\left(\mathrm{J}_{E_{R}}\right)$ \\
Pass & $<=$ Primary Threshold \\
Nominal Pass & $<=$ Secondary Threshold \\
Fail & $>$ Secondary Threshold
\end{tabular}

${ }^{5}$ With the notation used in this document, testing metrics $(M)$ are qualified with a subscripted tag (e.g., $M_{\text {size }}$ ). An italicized metric denotes the metric's value (e.g., $M_{\text {size }}$ ), while a non-italicized metric denotes the metric's function (e.g., $M_{\text {size }}\left(J_{E_{R}}\right)$ ). 
Table 7 - Lossy Compressed File Size: Reference Image Set Values and Thresholds

\begin{tabular}{|c|c|c|c|c|c|}
\hline $\begin{array}{c}\text { Image } \\
\qquad \#\end{array}$ & $\begin{array}{l}\text { Impression } \\
\text { Type }\end{array}$ & $\begin{array}{l}\text { Reference } \\
\text { Image File }\end{array}$ & $\begin{array}{c}\text { Reference } \\
\text { Measurement }\end{array}$ & $\begin{array}{l}<=\text { Primary } \\
\text { Threshold }\end{array}$ & $\begin{array}{c}<=\text { Secondary } \\
\text { Threshold }\end{array}$ \\
\hline & & & $\mathrm{M}_{\text {size }}\left(\mathrm{J}_{\mathrm{E}_{\mathrm{R}}}\right)$ & Pass & Nominal Pass \\
\hline 1 & Flat & S0011_1000_08P-ER.jp2 & 46436 & 48758 & 51080 \\
\hline 2 & Flat & S0187_1000_07P-ER.jp2 & 58587 & 61517 & 64446 \\
\hline 3 & Flat & S0201_1000_02P-ER.jp2 & 58816 & 61757 & 64698 \\
\hline 4 & Flat & S0417_1000_11-ER.jp2 & 133040 & 139692 & 146344 \\
\hline 5 & Flat & S0464_1000_09P-ER.jp2 & 40832 & 42874 & 44916 \\
\hline 6 & Flat & S0564_1000_03P-ER.jp2 & 53982 & 56682 & 59381 \\
\hline 7 & Flat & S0586_1000_10P-ER.jp2 & 53133 & 55790 & 58447 \\
\hline 8 & Flat & S0676_1000_12-ER.jp2 & 133270 & 139934 & 146597 \\
\hline 9 & Flat & S0704_1000_05P-ER.jp2 & 33586 & 35266 & 36945 \\
\hline 10 & Flat & S0730_1000_04P-ER.jp2 & 54870 & 57614 & 60357 \\
\hline 11 & Rolled & S0051_1000_05-ER.jp2 & 231870 & 243464 & 255057 \\
\hline 12 & Rolled & S0093_1000_01-ER.jp2 & 244423 & 256645 & 268866 \\
\hline 13 & Rolled & S0152_1000_06-ER.jp2 & 259630 & 272612 & 285593 \\
\hline 14 & Rolled & S0176_1000_02-ER.jp2 & 223879 & 235073 & 246267 \\
\hline 15 & Rolled & S0249_1000_08-ER.jp2 & 204796 & 215036 & 225276 \\
\hline 16 & Rolled & S0342_1000_09-ER.jp2 & 226139 & 237446 & 248753 \\
\hline 17 & Rolled & S0397_1000_04-ER.jp2 & 213167 & 223826 & 234484 \\
\hline 18 & Rolled & S0503_1000_07-ER.jp2 & 207617 & 217998 & 228379 \\
\hline 19 & Rolled & S0705_1000_03-ER.jp2 & 209527 & 220004 & 230480 \\
\hline 20 & Rolled & S0724_1000_10-ER.jp2 & 222856 & 233999 & 245142 \\
\hline 21 & Slap & S0139_1000_13-ER.jp2 & 603779 & 633968 & 664157 \\
\hline 22 & Slap & S0286_1000_13-ER.jp2 & 637109 & 668965 & 700820 \\
\hline 23 & Slap & S0304_1000_14-ER.jp2 & 620026 & 651028 & 682029 \\
\hline 24 & Slap & S0392_1000_13-ER.jp2 & 624604 & 655835 & 687065 \\
\hline 25 & Slap & S0465_1000_14-ER.jp2 & 619989 & 650989 & 681988 \\
\hline 26 & Slap & S0466_1000_13-ER.jp2 & 637003 & 668854 & 700704 \\
\hline 27 & Slap & S0641_1000_14-ER.jp2 & 596373 & 626192 & 656011 \\
\hline 28 & Slap & S0795_1000_14-ER.jp2 & 570751 & 599289 & 627827 \\
\hline 29 & Slap & S0817_1000_13-ER.jp2 & 637108 & 668964 & 700819 \\
\hline 30 & Slap & S0850_1000_14-ER.jp2 & 584161 & 613370 & 642578 \\
\hline
\end{tabular}


Table 8 - Lossless Compressed File Size: Reference Image Set Values and Thresholds

\begin{tabular}{|c|c|c|c|c|c|}
\hline $\begin{array}{c}\text { Image } \\
\quad \#\end{array}$ & $\begin{array}{c}\text { Impression } \\
\text { Type }\end{array}$ & $\begin{array}{l}\text { Reference } \\
\text { Image File }\end{array}$ & $\begin{array}{c}\text { Reference } \\
\text { Measurement }\end{array}$ & $\begin{array}{l}<=\text { Primary } \\
\text { Threshold }\end{array}$ & $\begin{array}{c}<=\text { Secondary } \\
\text { Threshold }\end{array}$ \\
\hline & & & $\mathrm{M}_{\text {size }}\left(\mathrm{J}_{\mathrm{E}_{R}}\right)$ & Pass & Nominal Pass \\
\hline 1 & Flat & S0011_1000_08P-LER.jp2 & 232773 & 244412 & 256051 \\
\hline 2 & Flat & S0187_1000_07P-LER.jp2 & 277215 & 291076 & 304937 \\
\hline 3 & Flat & S0201_1000_02P-LER.jp2 & 271663 & 285247 & 298830 \\
\hline 4 & Flat & S0417_1000_11-LER.jp2 & 626452 & 657775 & 689098 \\
\hline 5 & Flat & S0464_1000_09P-LER.jp2 & 198169 & 208078 & 217986 \\
\hline 6 & Flat & S0564_1000_03P-LER.jp2 & 277351 & 291219 & 305087 \\
\hline 7 & Flat & S0586_1000_10P-LER.jp2 & 273112 & 286768 & 300424 \\
\hline 8 & Flat & S0676_1000_12-LER.jp2 & 517174 & 543033 & 568892 \\
\hline 9 & Flat & S0704_1000_05P-LER.jp2 & 168205 & 176616 & 185026 \\
\hline 10 & Flat & S0730_1000_04P-LER.jp2 & 268649 & 282082 & 295514 \\
\hline 11 & Rolled & S0051_1000_05-LER.jp2 & 1064588 & 1117818 & 1171047 \\
\hline 12 & Rolled & S0093_1000_01-LER.jp2 & 1138901 & 1195847 & 1252792 \\
\hline 13 & Rolled & S0152_1000_06-LER.jp2 & 1234459 & 1296182 & 1357905 \\
\hline 14 & Rolled & S0176_1000_02-LER.jp2 & 1098514 & 1153440 & 1208366 \\
\hline 15 & Rolled & S0249_1000_08-LER.jp2 & 1003864 & 1054058 & 1104251 \\
\hline 16 & Rolled & S0342_1000_09-LER.jp2 & 1085240 & 1139502 & 1193764 \\
\hline 17 & Rolled & S0397_1000_04-LER.jp2 & 1019982 & 1070982 & 1121981 \\
\hline 18 & Rolled & S0503_1000_07-LER.jp2 & 978388 & 1027308 & 1076227 \\
\hline 19 & Rolled & S0705_1000_03-LER.jp2 & 953767 & 1001456 & 1049144 \\
\hline 20 & Rolled & S0724_1000_10-LER.jp2 & 1028500 & 1079925 & 1131350 \\
\hline 21 & Slap & S0139_1000_13-LER.jp2 & 2639358 & 2771326 & 2903294 \\
\hline 22 & Slap & S0286_1000_13-LER.jp2 & 3196022 & 3355824 & 3515625 \\
\hline 23 & Slap & S0304_1000_14-LER.jp2 & 2483632 & 2607814 & 2731996 \\
\hline 24 & Slap & S0392_1000_13-LER.jp2 & 2673289 & 2806954 & 2940618 \\
\hline 25 & Slap & S0465_1000_14-LER.jp2 & 2761384 & 2899454 & 3037523 \\
\hline 26 & Slap & S0466_1000_13-LER.jp2 & 2756295 & 2894110 & 3031925 \\
\hline 27 & Slap & S0641_1000_14-LER.jp2 & 2467987 & 2591387 & 2714786 \\
\hline 28 & Slap & S0795_1000_14-LER.jp2 & 2460837 & 2583879 & 2706921 \\
\hline 29 & Slap & S0817_1000_13-LER.jp2 & 2897104 & 3041960 & 3186815 \\
\hline 30 & Slap & S0850_1000_14-LER.jp2 & 2400488 & 2520513 & 2640537 \\
\hline
\end{tabular}




\subsubsection{Compressed File Structure and Metadata}

Table 9 - Compressed File Structure and Metadata - Criteria

\begin{tabular}{|c|c|c|c|}
\hline JPEG 2000 Box / Data Field & Value & Size (Bytes) & Hexadecima \\
\hline \multicolumn{4}{|l|}{ JPEG2000 Signature Box } \\
\hline Length & 12 & 4 & $0000000 \mathrm{C}$ \\
\hline Type & 'jP $<\mathrm{SP}>\langle\mathrm{SP}\rangle{ }^{\prime}$ & 4 & $6 A 502020$ \\
\hline Signature & $'<\mathrm{CR}><\mathrm{LF}><0 \times 87><\mathrm{LF}>{ }^{\prime}$ & 4 & OD0A 870A \\
\hline \multicolumn{4}{|l|}{ File Type Box } \\
\hline Length & 20 & 4 & 00000014 \\
\hline Type & 'ftyp' & 4 & 66747970 \\
\hline Brand (BR) & 'jp2<SP>' & 4 & $6 A 703220$ \\
\hline Minor Version (MinV) & 0 & 4 & 00000000 \\
\hline Compatibility List (CL) & 'jp2<SP>' & 4 & $6 A 703220$ \\
\hline \multicolumn{4}{|l|}{ JP2 Header Box (Superbox) } \\
\hline Length & 71 & 4 & 00000047 \\
\hline Type & 'jp2h' & 4 & $6 A 703268$ \\
\hline \multicolumn{4}{|l|}{ Image Header Box } \\
\hline Length & 22 & 4 & 00000016 \\
\hline Type & 'indr' & 4 & 69686472 \\
\hline Height & Image Area Height (pixels) & 4 & \\
\hline Width & Image Area Width (pixels) & 4 & \\
\hline Number of Components (NC) & 1 & 2 & 0001 \\
\hline Bit Depth minus 1 (BPC) & 7 (Corresponds to 8bpp) & 1 & 07 \\
\hline Compression Type (C) & 7 (JPEG2000) & 1 & 07 \\
\hline Colorspace Unknown (UnkC) & 0 & 1 & 00 \\
\hline Intellectual Property (IPR) & 0 & 1 & 00 \\
\hline \multicolumn{4}{|l|}{ Color Specification Box } \\
\hline Length & 15 & 4 & $0000000 \mathrm{~F}$ \\
\hline Type & 'colr' & 4 & $636 \mathrm{~F} 6 \mathrm{C} 72$ \\
\hline Method (METH) & 1 (Enumerated Colorspace) & 1 & 01 \\
\hline Precedence (PREC) & 0 (reserved) & 1 & 00 \\
\hline Approximation (APPROX) & 0 (reserved) & 1 & 00 \\
\hline Enumerated Colorspace (EnumCS) & 17 (grayscale) & 4 & 00000011 \\
\hline \multicolumn{4}{|l|}{ Resolution Box (Superbox) } \\
\hline Length & 26 & 4 & $0000001 \mathrm{~A}$ \\
\hline Type & 'res' & 4 & 72657320 \\
\hline \multicolumn{4}{|l|}{ Capture Resolution Box } \\
\hline Length & 18 & 4 & 00000012 \\
\hline Type & 'resc' & 4 & 72657363 \\
\hline Vertical Capture Resolution Numerator (VRcN) & $39370^{6}$ & 2 & 99CA \\
\hline Vertical Capture Resolution Denominator (VRcD) & 1 & 2 & 0001 \\
\hline Horizontal Capture Resolution Numerator (HRcN) & $39370^{6}$ & 2 & 99CA \\
\hline Horizontal Capture Resolution Denominator (HRcD) & 1 & 2 & 0001 \\
\hline Vertical Capture Resolution Exponent (VRdE) & 0 & 1 & 00 \\
\hline Horizontal Capture Resolution Exponent (HRdE) & 0 & 1 & 00 \\
\hline Contiguous Codestream Box & See section 4.1 of [NIST3] & & \\
\hline \multicolumn{4}{|l|}{ Identification and MetaData Box (Comment) } \\
\hline Marker Code & COM & 2 & FF64 \\
\hline Comment Length (Lcom) & 104 & 2 & 0068 \\
\hline Registration Value (Rcom) & 1 (ISO/IEC 8859-15 data) & 2 & 0001 \\
\hline Comment Contents & (see section 5.1 .3 ) & 100 & \\
\hline
\end{tabular}

${ }^{6}$ Values for VRcN and HrcN are specified here in pixels per meter according to [JPEG2K]. A value of 39370 corresponds to 1000 pixels per inch. 
This metric $\left(M_{\text {struct }}\right)$ evaluates the content of the JPEG 2000 file $\left(J_{E_{S}}\right)$ produced by the supplier's encoder. The compressed JPEG 2000 files must be strictly structured and populated with metadata as specified by Table 9 . Grades (in this case only a grade of Pass or Fail) are assessed based on the criteria in Table 10.

Table 10 - Compressed File Structure and Metadata - Grades

\begin{tabular}{|l|l|}
\hline Pass & $\mathrm{M}_{\text {struct }}\left(\mathrm{J}_{\mathrm{E}_{\mathrm{S}}}\right)$, Criteria in Table 9 strictly met \\
\hline Fail & $\mathrm{M}_{\text {struct }}\left(\mathrm{J}_{\mathrm{E}_{\mathrm{S}}}\right)$, Criteria in Table 9 is not strictly met \\
\hline
\end{tabular}

\subsubsection{Encoder Identification}

The Identification and Metadata Box is a special purpose comment box that can accommodate up to 100 bytes of structured ISO/IEC 8859-15 [ISO/IEC99] data. This data, also referred to as "comment contents," is used for certification and identification. The structure of the data is described in Table 11 along with sample identification data. The actual identification data must be obtained from NIST on a per-implementation basis. See Section 6 for information on how to obtain supplier identification data needed for this field.

Table 11 - Identification and Metadata Structure

\begin{tabular}{l|l|l|l}
\hline Data Segment Description & $\begin{array}{l}\text { Start } \\
\text { Position }\end{array}$ & Length & Example Contents \\
\hline Encoder Identification Tag & 0 & 7 & 'EnclD: ' \\
\hline Encoder Identification Data & 7 & 20 & 'CERT-SUBMISSION-0000' \\
Reserved Block Tag & 27 & 8 & 'Resvd: ' \\
Reserved Block Data & 35 & 65 & '41ab87823014dcb19113dfcd0902c569265270861a7f2cbdff148a38cc260675' \\
\hline
\end{tabular}

\subsection{Decoder Metrics}

The decoder metrics used in this testing are all image-based analyses. All the decoder metrics in this section apply to lossy compression, while only the first two (Image Pixel Dimension, and Altered Pixel Count) are applicable to evaluate the lossless compression mode.

\subsubsection{Image Pixel Dimensions}

This metric $\left(M_{d i m}\right)$ evaluates the pixel dimensions of a processed image fingerprint image after it has gone through an encoder plus decoder pathway (e.g., $\mathrm{P}_{\mathrm{E}_{S} \mathrm{D}_{\mathrm{R}}}$ ). The number of pixel columns and rows must be the same as that of the source reference fingerprint image, PsRC. Grades (in this case only a grade of Pass) are assessed based on the criteria in Table 12. The values of the pixel dimensions associated with the reference fingerprint image set are listed in Table 2.

Table 12 - Image Pixel Dimension - Grades

\begin{tabular}{ll}
\hline Pass & Pixel dimensions are the same, e.g., $M_{\text {dim }}\left(P_{E_{S} D_{R}}, P_{S R C}\right)$ \\
Fail & Pixel dimension are different
\end{tabular}




\subsubsection{Altered Pixel Count}

This metric $\left(M_{\text {altered }}\right)$ evaluates how many pixels have been altered in a processed fingerprint image after it has gone through an encoder plus decoder pathway (e.g., $\mathrm{P}_{\mathrm{E}_{S} \mathrm{D}_{\mathrm{S}}}$ ). The processed image pixels are compared with those in the source reference fingerprint image $\left(\mathrm{P}_{\mathrm{SRC}}\right)$ and each pixel changed is tallied.

\subsubsection{Altered Pixel Count (for Lossy Compression)}

Given a source image, $\boldsymbol{l}^{\varnothing}$ (i.e., $\mathrm{P}_{\mathrm{SRC}}$ ), and the corresponding processed image, $\mid \delta\left(\right.$ e.g., $\mathrm{P}_{\mathrm{ES}_{\mathrm{S}} \mathrm{D}_{\mathrm{S}}}$ ), the pixel values of the difference image, $\boldsymbol{D}$, can be computed as

$$
D_{i, j}=\left|I_{i, j}^{\emptyset}-I_{i, j}^{\delta}\right|,
$$

where $i=1 . . R$, and $j=1$.. $C, R=$ number of pixel rows and $C=$ number of pixel columns.

Given the difference image, $\boldsymbol{D}$, we construct a bi-level image, $\boldsymbol{B}$, to which we assign binary values as

$$
B_{i, j}=\left\{\begin{array}{ll}
0 & D_{i, j}=0 \\
1 & D_{i, j} \neq 0
\end{array} .\right.
$$

We then count the total number of changed pixels as

$$
M_{\text {altered }}=\sum_{i=1}^{R} \sum_{j=1}^{C} B_{i, j} .
$$

The tolerances used for this metric are based on a margin computed between measurements made at both 10:1 and 12:1 using the NIST reference CODEC. (Recall the earlier discussion in Section 3.1 that human examiners observed forensicallyrelevant degradation between these two compression levels when the reference fingerprint image set was used.) In this case the total altered pixels from a processed image (e.g., $\mathrm{P}_{\mathrm{E}_{s} \mathrm{D}_{\mathrm{S}}}$ ) are compared to a margin computed as the difference between the total altered pixels from its source image $\left(P_{\text {SRC }}\right)$ processed through the pathway of the reference encoder and reference decoder $\left(P_{E_{R} D_{R}}\right)$ using the two compression levels 12:1 and 10:1. In the example used here, we are evaluating a processed image having gone through the pathway of the supplier's encoder and supplier's decoder $\left(E_{s} D_{s}\right)$. Grades are assessed based on measures set according to Table 13. The thresholds defined in Table 13 are computed across the reference fingerprint image set and listed in Table 14.

Table 13 - Altered Pixel Count (Lossy): Measures and Grades

\begin{tabular}{ll}
\hline Value Being Graded (e.g.) & $M_{\text {altered }}\left(10: 1, P_{E_{S} D_{S}}, P_{S R C}\right)$ \\
Reference Measurement @ 10:1 & $M_{\text {altered }}\left(10: 1, P_{E_{R} D_{R}}, P_{S R C}\right)$ \\
\hline Reference Measurement @ 12:1 & $M_{\text {altered }}\left(12: 1, P_{E_{R} D_{R}}, P_{S R C}\right)$ \\
\hline Margin & $M_{\text {altered }}\left(12: 1, P_{E_{R} D_{R}}, P_{S R C}\right)-M_{\text {altered }}\left(10: 1, P_{E_{R} D_{R}}, P_{S R C}\right)$ \\
\hline Primary Tolerance & $25 \%$ Margin \\
\hline Secondary Tolerance & $50 \%$ Margin \\
\hline Primary Threshold & $M_{\text {altered }}\left(10: 1, P_{E_{R} D_{R}}, P_{S R C}\right)+$ Primary Tolerance \\
\hline Secondary Threshold & $M_{\text {altered }}\left(10: 1, P_{E_{R} D_{R}}, P_{S R C}\right)+$ Secondary Tolerance \\
\hline Gold (Pass) & $<M_{\text {altered }}\left(10: 1, P_{E_{R} D_{R}}, P_{S R C}\right)$ \\
Pass & $<=$ Primary Threshold \\
Nominal Pass & $<=$ Secondary Threshold \\
Fail & $>$ Secondary Threshold
\end{tabular}


Table 14 - Altered Pixel Count (Lossy): Reference Image Set Values and Thresholds

\begin{tabular}{|c|c|c|c|c|c|c|}
\hline \multirow[t]{2}{*}{$\begin{array}{c}\text { Image } \\
\quad \#\end{array}$} & \multirow[t]{2}{*}{$\begin{array}{l}\text { Impression } \\
\text { Type }\end{array}$} & \multirow[t]{2}{*}{$\begin{array}{l}\text { Reference } \\
\text { Image File }\end{array}$} & \multicolumn{2}{|c|}{$\begin{array}{c}\text { Reference } \\
\text { Measurements }\end{array}$} & \multirow{2}{*}{$\begin{array}{c}<=\text { Primary } \\
\text { Threshold } \\
\text { Pass }\end{array}$} & \multirow{2}{*}{$\begin{array}{c}<=\text { Secondary } \\
\text { Threshold } \\
\text { Nominal Pass }\end{array}$} \\
\hline & & & $10: 1$ & $12: 1$ & & \\
\hline 1 & Flat & S0011_1000_08P-SRC.pgm & 391852 & 397494 & 393263 & 394673 \\
\hline 2 & Flat & S0187_1000_07P-SRC.pgm & 478065 & 490211 & 481102 & 484138 \\
\hline 3 & Flat & S0201_1000_02P-SRC.pgm & 469514 & 481034 & 472394 & 475274 \\
\hline 4 & Flat & S0417_1000_11-SRC.pgm & 1092182 & 1118798 & 1098836 & 1105490 \\
\hline 5 & Flat & S0464_1000_09P-SRC.pgm & 338666 & 346175 & 340543 & 342421 \\
\hline 6 & Flat & S0564_1000_03P-SRC.pgm & 461621 & 468273 & 463284 & 464947 \\
\hline 7 & Flat & S0586_1000_10P-SRC.pgm & 455618 & 460669 & 456881 & 458144 \\
\hline 8 & Flat & S0676_1000_12-SRC.pgm & 943253 & 979479 & 952310 & 961366 \\
\hline 9 & Flat & S0704_1000_05P-SRC.pgm & 287277 & 290954 & 288196 & 289116 \\
\hline 10 & Flat & S0730_1000_04P-SRC.pgm & 459019 & 467160 & 461054 & 463090 \\
\hline 11 & Rolled & S0051_1000_05-SRC.pgm & 1860517 & 1911125 & 1873169 & 1885821 \\
\hline 12 & Rolled & S0093_1000_01-SRC.pgm & 1983255 & 2036445 & 1996553 & 2009850 \\
\hline 13 & Rolled & S0152_1000_06-SRC.pgm & 2142413 & 2192629 & 2154967 & 2167521 \\
\hline 14 & Rolled & S0176_1000_02-SRC.pgm & 1875992 & 1914548 & 1885631 & 1895270 \\
\hline 15 & Rolled & S0249_1000_08-SRC.pgm & 1708272 & 1741492 & 1716577 & 1724882 \\
\hline 16 & Rolled & S0342_1000_09-SRC.pgm & 1878842 & 1917308 & 1888459 & 1898075 \\
\hline 17 & Rolled & S0397_1000_04-SRC.pgm & 1755274 & 1800625 & 1766612 & 1777950 \\
\hline 18 & Rolled & S0503_1000_07-SRC.pgm & 1699051 & 1744889 & 1710511 & 1721970 \\
\hline 19 & Rolled & S0705_1000_03-SRC.pgm & 1679192 & 1730520 & 1692024 & 1704856 \\
\hline 20 & Rolled & S0724_1000_10-SRC.pgm & 1803623 & 1853564 & 1816108 & 1828594 \\
\hline 21 & Slap & S0139_1000_13-SRC.pgm & 4667198 & 4823830 & 4706356 & 4745514 \\
\hline 22 & Slap & S0286_1000_13-SRC.pgm & 5412337 & 5495599 & 5433153 & 5453968 \\
\hline 23 & Slap & S0304_1000_14-SRC.pgm & 4418345 & 4558482 & 4453379 & 4488414 \\
\hline 24 & Slap & S0392_1000_13-SRC.pgm & 4685112 & 4856213 & 4727887 & 4770663 \\
\hline 25 & Slap & S0465_1000_14-SRC.pgm & 4870952 & 5010692 & 4905887 & 4940822 \\
\hline 26 & Slap & S0466_1000_13-SRC.pgm & 4852427 & 5026205 & 4895872 & 4939316 \\
\hline 27 & Slap & S0641_1000_14-SRC.pgm & 4426848 & 4566900 & 4461861 & 4496874 \\
\hline 28 & Slap & S0795_1000_14-SRC.pgm & 4393716 & 4526321 & 4426867 & 4460019 \\
\hline 29 & Slap & S0817_1000_13-SRC.pgm & 5095847 & 5244884 & 5133106 & 5170366 \\
\hline 30 & Slap & S0850_1000_14-SRC.pgm & 4343816 & 4482193 & 4378410 & 4413005 \\
\hline
\end{tabular}

\subsubsection{Altered Pixel Count (for Lossless Compression)}

The criteria for evaluating lossless compression in regard to altered image pixels are straight forward. For the lossless mode of a supplier's CODEC to Pass there must be zero altered pixels between the source image $\left(\mathrm{P}_{\mathrm{SRC}}\right)$ and the processed image

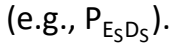

Table 15 - Altered Pixel Count (Lossless) - Grades

\begin{tabular}{ll}
\hline Pass & e.g., $M_{\text {altered }}\left(P_{S R C}, P_{E_{S} D_{S}}\right)$ must equal 0 \\
Fail & $M_{\text {altered }}\left(P_{S R C}, P_{E_{S} D_{S}}\right)$ does not equal 0
\end{tabular}




\subsubsection{Peak Pixel Difference}

This metric $\left(M_{\text {peak }}\right)$ evaluates the pixel incurring the maximum gray level change comparing a processed image (e.g., $\left.\mathrm{P}_{\mathrm{E}_{\mathrm{s}} \mathrm{D}_{\mathrm{S}}}\right)$ to its source image (PSR). Given the absolute image difference, $\boldsymbol{D}$, from equation (1), the peak difference is then

$$
M_{\text {peak }}=\max \left(D_{\mathrm{i}, \mathrm{j}}\right)
$$

The tolerance used for this metric is based on a margin computed between measurements made at both 10:1 and 12:1 using the NIST reference CODEC. In this case the peak pixel difference from a processed image (e.g., $\mathrm{P}_{\mathrm{E}_{\mathrm{S}} \mathrm{D}_{\mathrm{S}}}$ ) is compared to the peak pixel difference from its source image $\left(\mathrm{P}_{\mathrm{SRC}}\right)$ processed through the pathway of the reference encoder and reference decoder $\left(P_{E_{R} D_{R}}\right)$ using 12:1 compression. For a supplier's CODEC to Pass the peak pixel difference of its processed image must be less than or equal to the maximum reference measurement between 10:1 and 12:1. Grades are assessed based on measures set according to Table 16. The threshold defined in Table 16 is computed across the reference fingerprint image set and listed in Table 17.

Table 16 - Peak Pixel Difference: Measures and Grades

\begin{tabular}{ll}
\hline Value Being Graded (e.g.) & $\mathrm{M}_{\text {peak }}\left(10: 1, \mathrm{P}_{\mathrm{E}_{S} D_{S}}, \mathrm{P}_{\mathrm{SRC}}\right)$ \\
\hline Reference Measurement @ 10:1 & $\mathrm{M}_{\text {peak }}\left(10: 1, \mathrm{P}_{\mathrm{E}_{R} D_{R}}, \mathrm{P}_{\mathrm{SRC}}\right)$ \\
\hline Reference Measurement @ 12:1 & $\mathrm{M}_{\text {peak }}\left(12: 1, \mathrm{P}_{\mathrm{E}_{R} D_{R}}, \mathrm{P}_{\mathrm{SRC}}\right)$ \\
\hline Primary Threshold & $\max ($ \\
& $\mathrm{M}_{\text {peak }}\left(10: 1, \mathrm{P}_{\left.\mathrm{E}_{\mathrm{R}} \mathrm{D}_{\mathrm{R}}, \mathrm{P}_{\mathrm{SRC}}\right),}\right.$ \\
& $\left.\mathrm{M}_{\text {peak }}\left(12: 1, \mathrm{P}_{\mathrm{E}_{\mathrm{R}} \mathrm{D}_{R}}, \mathrm{P}_{\mathrm{SRC}}\right)\right)$ \\
\hline Pass & $<=$ Primary Threshold \\
Fail & $>$ Primary Threshold
\end{tabular}


Table 17 - Peak Pixel Difference: Reference Image Set Values and Threshold

\begin{tabular}{|c|c|c|c|c|c|}
\hline \multirow[t]{2}{*}{$\begin{array}{c}\text { Image } \\
\#\end{array}$} & \multirow[t]{2}{*}{$\begin{array}{c}\text { Impression } \\
\text { Type }\end{array}$} & \multirow[t]{2}{*}{$\begin{array}{l}\text { Reference } \\
\text { Image File }\end{array}$} & \multicolumn{2}{|c|}{$\begin{array}{c}\text { Reference } \\
\text { Measurements }\end{array}$} & \multirow{2}{*}{$\begin{array}{c}<=\text { Primary } \\
\text { Threshold } \\
\text { Pass }\end{array}$} \\
\hline & & & $10: 1$ & $12: 1$ & \\
\hline 1 & Flat & S0011_1000_08P-SRC.pgm & 20 & 22 & 22 \\
\hline 2 & Flat & S0187_1000_07P-SRC.pgm & 19 & 19 & 19 \\
\hline 3 & Flat & S0201_1000_02P-SRC.pgm & 14 & 15 & 15 \\
\hline 4 & Flat & S0417_1000_11-SRC.pgm & 17 & 18 & 18 \\
\hline 5 & Flat & S0464_1000_09P-SRC.pgm & 15 & 17 & 17 \\
\hline 6 & Flat & S0564_1000_03P-SRC.pgm & 26 & 25 & 26 \\
\hline 7 & Flat & S0586_1000_10P-SRC.pgm & 18 & 22 & 22 \\
\hline 8 & Flat & S0676_1000_12-SRC.pgm & 9 & 10 & 10 \\
\hline 9 & Flat & S0704_1000_05P-SRC.pgm & 23 & 27 & 27 \\
\hline 10 & Flat & S0730_1000_04P-SRC.pgm & 18 & 21 & 21 \\
\hline 11 & Rolled & S0051_1000_05-SRC.pgm & 16 & 18 & 18 \\
\hline 12 & Rolled & S0093_1000_01-SRC.pgm & 18 & 20 & 20 \\
\hline 13 & Rolled & S0152_1000_06-SRC.pgm & 18 & 19 & 19 \\
\hline 14 & Rolled & S0176_1000_02-SRC.pgm & 18 & 25 & 25 \\
\hline 15 & Rolled & S0249_1000_08-SRC.pgm & 19 & 26 & 26 \\
\hline 16 & Rolled & S0342_1000_09-SRC.pgm & 17 & 19 & 19 \\
\hline 17 & Rolled & S0397_1000_04-SRC.pgm & 15 & 19 & 19 \\
\hline 18 & Rolled & S0503_1000_07-SRC.pgm & 16 & 18 & 18 \\
\hline 19 & Rolled & S0705_1000_03-SRC.pgm & 15 & 23 & 23 \\
\hline 20 & Rolled & S0724_1000_10-SRC.pgm & 15 & 16 & 16 \\
\hline 21 & Slap & S0139_1000_13-SRC.pgm & 16 & 21 & 21 \\
\hline 22 & Slap & S0286_1000_13-SRC.pgm & 33 & 33 & 33 \\
\hline 23 & Slap & S0304_1000_14-SRC.pgm & 15 & 21 & 21 \\
\hline 24 & Slap & S0392_1000_13-SRC.pgm & 17 & 25 & 25 \\
\hline 25 & Slap & S0465_1000_14-SRC.pgm & 19 & 20 & 20 \\
\hline 26 & Slap & S0466_1000_13-SRC.pgm & 18 & 21 & 21 \\
\hline 27 & Slap & S0641_1000_14-SRC.pgm & 16 & 18 & 18 \\
\hline 28 & Slap & S0795_1000_14-SRC.pgm & 16 & 18 & 18 \\
\hline 29 & Slap & S0817_1000_13-SRC.pgm & 25 & 26 & 26 \\
\hline 30 & Slap & S0850_1000_14-SRC.pgm & 14 & 15 & 15 \\
\hline
\end{tabular}




\subsubsection{Image Mean Squared Difference}

This metric $\left(M_{M S D}\right)$ uses the Image Mean Squared Difference (MSD) - a commonly used image fidelity attribute - to evaluate the amount of gray level pixel change across the entire processed fingerprint image after it has gone through an encoder plus decoder pathway (e.g., $\mathrm{P}_{E_{S} D_{S}}$ ). Given a source image, $\boldsymbol{l}^{\varnothing}$ (i.e., $\mathrm{P}_{S R C}$ ), and a processed image, $\boldsymbol{l}^{\delta}\left(\right.$ e.g., $\left.\mathrm{P}_{\mathrm{E}_{S} \mathrm{D}_{S}}\right)$, the image mean squared difference is defined as

$$
M_{M S D}=\frac{\sum_{i=1}^{R} \sum_{j=1}^{C}\left(I_{i, j}^{\emptyset}-I_{i, j}^{\delta}\right)^{2}}{R C},
$$

where $i$ and $j$ are indices to image pixels, and $R$ and $C$ are numbers of image rows and columns, respectively. Note that the source image and processed image must be equal in dimensions in order to apply this metric.

The tolerances used for this metric are based on a margin computed between measurements made at both 10:1 and 12:1 using the NIST reference CODEC. In this case the MSD from a processed image (e.g., $P_{E_{S} D_{S}}$ ) are compared to a margin computed as the difference between the MSD from its source image ( $P_{S R C}$ ) processed through the pathway of the reference encoder and reference decoder $\left(P_{E_{R} D_{R}}\right)$ using the two compression levels 12:1 and 10:1. In the example used here, we are evaluating a processed image having gone through the pathway of the supplier's encoder and supplier's decoder $\left(E_{s} D_{s}\right)$. Grades are assessed based on measures set according to Table 18. The thresholds defined in Table 18 are computed across the reference fingerprint image set and listed in Table 19.

Table 18 - Image Mean Squared Difference: Measures and Grades

\begin{tabular}{lll}
\hline Value Being Graded (e.g.) & $M_{M S D}\left(10: 1, P_{E_{S} D_{S}}, P_{S R C}\right)$ \\
\hline Reference Measurement @ 10:1 & $M_{M S D}\left(10: 1, P_{E_{R} D_{R}}, P_{S R C}\right)$ \\
\hline Reference Measurement @ 12:1 & $M_{M S D}\left(12: 1, P_{E_{R} D_{R}}, P_{S R C}\right)$ \\
\hline Margin & $M_{M S D}\left(12: 1, P_{E_{R} D_{R}}, P_{S R C}\right)-M_{M S D}\left(10: 1, P_{E_{R} D_{R}}, P_{S R C}\right)$ \\
\hline Primary Tolerance & $25 \%$ Margin \\
\hline Secondary Tolerance & $50 \%$ Margin \\
\hline Primary Threshold & $M_{M S D}\left(10: 1, P_{E_{R} D_{R}}, P_{S R C}\right)+$ Primary Tolerance \\
\hline Secondary Threshold & $M_{M S D}\left(10: 1, P_{E_{R} D_{R}}, P_{S R C}\right)+$ Secondary Tolerance \\
\hline Gold (Pass) & $<M_{M S D}\left(10: 1, P_{E_{R} D_{R}}, P_{S R C}\right)$ \\
\hline Pass & $<=$ Primary Threshold \\
\hline Nominal Pass & $<=$ Secondary Threshold \\
\hline Fail & $>$ Secondary Threshold \\
\hline
\end{tabular}


Table 19 - Image Mean Squared Difference: Reference Image Set Values and Thresholds

\begin{tabular}{|c|c|c|c|c|c|c|}
\hline \multirow[t]{2}{*}{$\begin{array}{c}\text { Image } \\
\#\end{array}$} & \multirow[t]{2}{*}{$\begin{array}{l}\text { Impression } \\
\text { Type }\end{array}$} & \multirow[t]{2}{*}{$\begin{array}{l}\text { Reference } \\
\text { Image File }\end{array}$} & \multicolumn{2}{|c|}{$\begin{array}{c}\text { Reference } \\
\text { Measurements }\end{array}$} & \multirow{2}{*}{$\begin{array}{c}<=\text { Primary } \\
\text { Threshold } \\
\text { Pass }\end{array}$} & \multirow{2}{*}{$\begin{array}{c}<=\text { Secondary } \\
\text { Threshold } \\
\text { Nominal Pass }\end{array}$} \\
\hline & & & $10: 1$ & $12: 1$ & & \\
\hline 1 & Flat & S0011_1000_08P-SRC.pgm & 7.566 & 9.944 & 8.161 & 8.755 \\
\hline 2 & Flat & S0187_1000_07P-SRC.pgm & 5.420 & 6.776 & 5.759 & 6.098 \\
\hline 3 & Flat & S0201_1000_02P-SRC.pgm & 4.539 & 5.782 & 4.850 & 5.161 \\
\hline 4 & Flat & S0417_1000_11-SRC.pgm & 5.799 & 7.269 & 6.167 & 6.534 \\
\hline 5 & Flat & S0464_1000_09P-SRC.pgm & 6.536 & 8.101 & 6.927 & 7.319 \\
\hline 6 & Flat & S0564_1000_03P-SRC.pgm & 9.122 & 11.731 & 9.774 & 10.427 \\
\hline 7 & Flat & S0586_1000_10P-SRC.pgm & 8.660 & 11.158 & 9.285 & 9.909 \\
\hline 8 & Flat & S0676_1000_12-SRC.pgm & 1.977 & 2.330 & 2.065 & 2.154 \\
\hline 9 & Flat & S0704_1000_05P-SRC.pgm & 8.521 & 11.239 & 9.201 & 9.880 \\
\hline 10 & Flat & S0730_1000_04P-SRC.pgm & 6.793 & 8.852 & 7.308 & 7.823 \\
\hline 11 & Rolled & S0051_1000_05-SRC.pgm & 4.765 & 6.090 & 5.096 & 5.428 \\
\hline 12 & Rolled & S0093_1000_01-SRC.pgm & 5.233 & 6.626 & 5.581 & 5.930 \\
\hline 13 & Rolled & S0152_1000_06-SRC.pgm & 5.965 & 7.427 & 6.331 & 6.696 \\
\hline 14 & Rolled & S0176_1000_02-SRC.pgm & 6.589 & 8.005 & 6.943 & 7.297 \\
\hline 15 & Rolled & S0249_1000_08-SRC.pgm & 6.715 & 8.493 & 7.160 & 7.604 \\
\hline 16 & Rolled & S0342_1000_09-SRC.pgm & 6.289 & 7.733 & 6.650 & 7.011 \\
\hline 17 & Rolled & S0397_1000_04-SRC.pgm & 5.896 & 7.271 & 6.240 & 6.584 \\
\hline 18 & Rolled & S0503_1000_07-SRC.pgm & 5.553 & 6.936 & 5.899 & 6.245 \\
\hline 19 & Rolled & S0705_1000_03-SRC.pgm & 4.756 & 6.131 & 5.100 & 5.444 \\
\hline 20 & Rolled & S0724_1000_10-SRC.pgm & 4.924 & 6.252 & 5.256 & 5.588 \\
\hline 21 & Slap & S0139_1000_13-SRC.pgm & 3.607 & 4.808 & 3.907 & 4.208 \\
\hline 22 & Slap & S0286_1000_13-SRC.pgm & 8.383 & 11.081 & 9.058 & 9.732 \\
\hline 23 & Slap & S0304_1000_14-SRC.pgm & 2.390 & 3.149 & 2.580 & 2.770 \\
\hline 24 & Slap & S0392_1000_13-SRC.pgm & 3.071 & 4.012 & 3.306 & 3.542 \\
\hline 25 & Slap & S0465_1000_14-SRC.pgm & 4.143 & 5.449 & 4.470 & 4.796 \\
\hline 26 & Slap & S0466_1000_13-SRC.pgm & 3.350 & 4.457 & 3.627 & 3.904 \\
\hline 27 & Slap & S0641_1000_14-SRC.pgm & 2.603 & 3.319 & 2.782 & 2.961 \\
\hline 28 & Slap & S0795_1000_14-SRC.pgm & 3.282 & 4.123 & 3.492 & 3.703 \\
\hline 29 & Slap & S0817_1000_13-SRC.pgm & 4.818 & 6.212 & 5.167 & 5.515 \\
\hline 30 & Slap & S0850_1000_14-SRC.pgm & 2.596 & 3.266 & 2.764 & 2.931 \\
\hline
\end{tabular}




\subsubsection{Spectral Image Root Mean Squared Difference}

Developed initially as a method to screen fingerprint databases for non-fingerprint images, segmentation errors, or mislabeled sample rates, the Spectral Image Validation Verification (SIVV) metric [LIBERT] provides a comparatively straightforward method by which to assess the frequency structure of an image. Pairwise display of the SIVV signals of source and lossy compressed images enables summary visualization of the effects of compression across the composition frequency spectrum of the image as shown in Figure 2. As a 1-dimensional representation of a 2-dimensional Fourier spectrum, the SIVV metric applied to a fingerprint image exhibits a peak corresponding to the frequency of the ridge spacing (as shown within the circled region of the figure). In this example, the figure compares the SIVV signals of a source image and a JPEG 2000 processed image. Note the loss or gain of power over various frequencies.

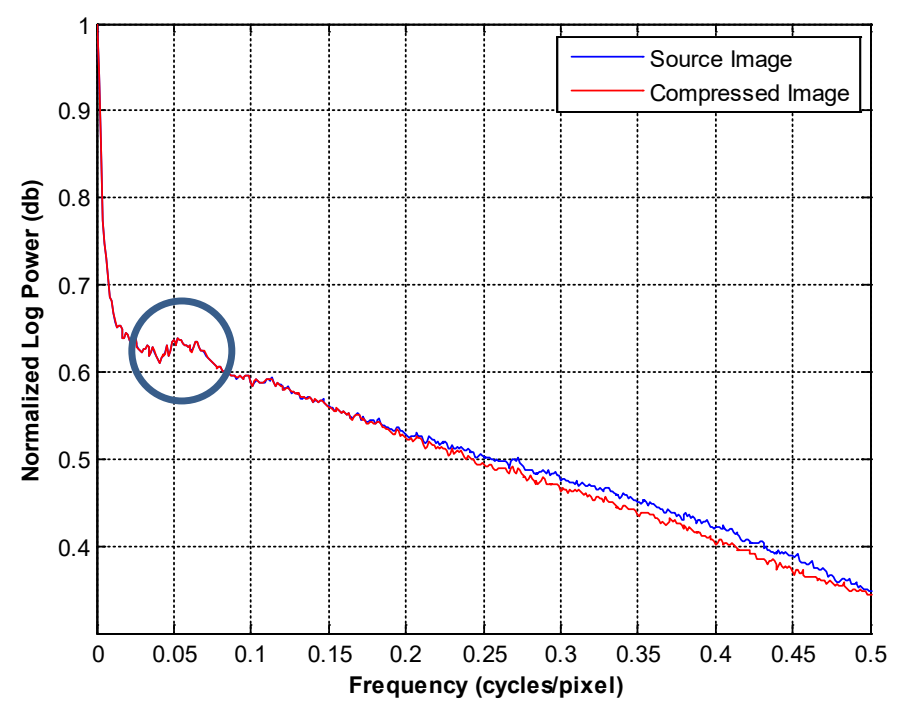

Figure 2 - SIVV signal of a fingerprint image before and after JPEG 2000 lossy compression

The metric $\left(M_{S I V V}\right)$ evaluates the amount of image frequency change in a processed fingerprint image after it has gone through an encoder plus decoder pathway (e.g., $\left.\mathrm{P}_{\mathrm{Es}_{\mathrm{s}}}\right)$. The processed image frequency profile is compared with that of the source reference fingerprint image $\left(\mathrm{P}_{\mathrm{SRC}}\right)$.

Given a source image, $l^{\varnothing}$ (i.e., $\mathrm{P}_{\mathrm{SRC}}$ ), and the subsequently processed image, $\boldsymbol{I}^{\delta}\left(\right.$ e.g., $\mathrm{P}_{\mathrm{ES}_{\mathrm{S}} \mathrm{D}}$ ), corresponding SIVV signal vectors are denoted $\mathbf{s}^{\varnothing}$ and $\mathbf{s}^{\delta}$. The Root Mean Squared Difference (RMSD) is used to quantify the total amount of image frequency change between the two signals:

$$
M_{\text {SIVV }}=\sqrt{\frac{\sum_{i=1}^{n}\left(s_{i}^{\emptyset}-s_{i}^{\delta}\right)^{2}}{n}},
$$

where $n=\left|\mathbf{s}^{\varnothing}\right|=\left|\mathbf{s}^{\delta}\right|$ (i.e., the lengths of the signal vectors).

The tolerances used for this metric are based on a margin computed between measurements made at both 10:1 and 12:1 using the NIST reference CODEC. In this case the total change in power at all image frequencies from a processed image (e.g., $\mathrm{P}_{\mathrm{E}_{s} \mathrm{D}_{\mathrm{S}}}$ ) are compared to a margin computed as the difference between the total change in power at corresponding frequencies from its source image $\left(\mathrm{P}_{\mathrm{SRC}}\right)$ processed through the pathway of the reference encoder and reference decoder $\left(P_{E_{R} D_{R}}\right)$ using the two compression levels 12:1 and 10:1. In the example used here, we are evaluating a processed image having gone through the pathway of the supplier's encoder and supplier's decoder $\left(E_{s} D_{s}\right)$. Grades are assessed based on measures set according to Table 20. The thresholds defined in Table 20 are computed across the reference fingerprint image set and listed in Table 21. 
Table 20 - Spectral Image Root Mean Squared Difference: Measures and Grades

\begin{tabular}{ll}
\hline Value Being Graded (e.g.) & $\mathrm{M}_{\mathrm{SIVV}}\left(10: 1, \mathrm{P}_{\mathrm{E}_{S} D_{S}}, \mathrm{P}_{\mathrm{SRC}}\right)$ \\
Reference Measurement @ 10:1 & $\mathrm{M}_{\mathrm{SIVV}}\left(10: 1, \mathrm{P}_{\mathrm{E}_{R} D_{R}}, \mathrm{P}_{\mathrm{SRC}}\right)$ \\
\hline Reference Measurement @ 12:1 & $\mathrm{M}_{\mathrm{SIVV}}\left(12: 1, \mathrm{P}_{\mathrm{E}_{R} D_{R}}, \mathrm{P}_{\mathrm{SRC}}\right)$ \\
\hline Margin & $\mathrm{M}_{\mathrm{SIVV}}\left(12: 1, \mathrm{P}_{\mathrm{E}_{R} D_{R}}, \mathrm{P}_{\mathrm{SRC}}\right)-\mathrm{M}_{\mathrm{SIVV}}\left(10: 1, \mathrm{P}_{\mathrm{E}_{R} D_{R}}, \mathrm{P}_{\mathrm{SRC}}\right)$ \\
\hline Primary Tolerance & $25 \%$ Margin \\
\hline Secondary Tolerance & $50 \%$ Margin \\
\hline Primary Threshold & $\mathrm{M}_{\mathrm{SIVV}}\left(10: 1, \mathrm{P}_{\mathrm{E}_{R} D_{R}}, \mathrm{P}_{\mathrm{SRC}}\right)+$ Primary Tolerance \\
\hline Secondary Threshold & $\mathrm{M}_{\mathrm{SIVV}}\left(10: 1, \mathrm{P}_{\mathrm{E}_{R} D_{R}}, \mathrm{P}_{\mathrm{SRC}}\right)+$ Secondary Tolerance \\
\hline Gold (Pass) & $<\mathrm{M}_{\mathrm{SIVV}}\left(10: 1, \mathrm{P}_{\mathrm{E}_{R} D_{R}}, \mathrm{P}_{\mathrm{SRC}}\right)$ \\
\hline Pass & $<=$ Primary Threshold \\
Nominal Pass & $<=$ Secondary Threshold \\
Fail & $>$ Secondary Threshold
\end{tabular}


Table 21 - Spectral Image Root Mean Squared Difference: Reference Image Set Values and Thresholds

\begin{tabular}{|c|c|c|c|c|c|c|}
\hline \multirow[t]{2}{*}{$\begin{array}{c}\text { Image } \\
\#\end{array}$} & \multirow[t]{2}{*}{$\begin{array}{l}\text { Impression } \\
\text { Type }\end{array}$} & \multirow[t]{2}{*}{$\begin{array}{l}\text { Reference } \\
\text { Image File }\end{array}$} & \multicolumn{2}{|c|}{$\begin{array}{c}\text { Reference } \\
\text { Measurements }\end{array}$} & \multirow{2}{*}{$\begin{array}{c}<=\text { Primary } \\
\text { Threshold } \\
\text { Pass }\end{array}$} & \multirow{2}{*}{$\begin{array}{c}<=\text { Secondary } \\
\text { Threshold } \\
\text { Nominal Pass }\end{array}$} \\
\hline & & & $10: 1$ & $12: 1$ & & \\
\hline 1 & Flat & S0011_1000_08P-SRC.pgm & 0.0049 & 0.0068 & 0.0054 & 0.0059 \\
\hline 2 & Flat & S0187_1000_07P-SRC.pgm & 0.0057 & 0.0079 & 0.0063 & 0.0068 \\
\hline 3 & Flat & S0201_1000_02P-SRC.pgm & 0.0068 & 0.0102 & 0.0077 & 0.0085 \\
\hline 4 & Flat & S0417_1000_11-SRC.pgm & 0.0040 & 0.0051 & 0.0043 & 0.0046 \\
\hline 5 & Flat & S0464_1000_09P-SRC.pgm & 0.0066 & 0.0073 & 0.0068 & 0.0070 \\
\hline 6 & Flat & S0564_1000_03P-SRC.pgm & 0.0058 & 0.0120 & 0.0074 & 0.0089 \\
\hline 7 & Flat & S0586_1000_10P-SRC.pgm & 0.0044 & 0.0070 & 0.0051 & 0.0057 \\
\hline 8 & Flat & S0676_1000_12-SRC.pgm & 0.0077 & 0.0086 & 0.0079 & 0.0082 \\
\hline 9 & Flat & S0704_1000_05P-SRC.pgm & 0.0094 & 0.0166 & 0.0112 & 0.0130 \\
\hline 10 & Flat & S0730_1000_04P-SRC.pgm & 0.0041 & 0.0094 & 0.0054 & 0.0068 \\
\hline 11 & Rolled & S0051_1000_05-SRC.pgm & 0.0080 & 0.0103 & 0.0086 & 0.0092 \\
\hline 12 & Rolled & S0093_1000_01-SRC.pgm & 0.0109 & 0.0136 & 0.0116 & 0.0123 \\
\hline 13 & Rolled & S0152_1000_06-SRC.pgm & 0.0064 & 0.0079 & 0.0068 & 0.0072 \\
\hline 14 & Rolled & S0176_1000_02-SRC.pgm & 0.0095 & 0.0110 & 0.0099 & 0.0103 \\
\hline 15 & Rolled & S0249_1000_08-SRC.pgm & 0.0071 & 0.0141 & 0.0089 & 0.0106 \\
\hline 16 & Rolled & S0342_1000_09-SRC.pgm & 0.0078 & 0.0094 & 0.0082 & 0.0086 \\
\hline 17 & Rolled & S0397_1000_04-SRC.pgm & 0.0094 & 0.0108 & 0.0098 & 0.0101 \\
\hline 18 & Rolled & S0503_1000_07-SRC.pgm & 0.0087 & 0.0104 & 0.0091 & 0.0096 \\
\hline 19 & Rolled & S0705_1000_03-SRC.pgm & 0.0104 & 0.0128 & 0.0110 & 0.0116 \\
\hline 20 & Rolled & S0724_1000_10-SRC.pgm & 0.0100 & 0.0123 & 0.0106 & 0.0112 \\
\hline 21 & Slap & S0139_1000_13-SRC.pgm & 0.0028 & 0.0035 & 0.0030 & 0.0032 \\
\hline 22 & Slap & S0286_1000_13-SRC.pgm & 0.0034 & 0.0047 & 0.0037 & 0.0041 \\
\hline 23 & Slap & S0304_1000_14-SRC.pgm & 0.0040 & 0.0064 & 0.0046 & 0.0052 \\
\hline 24 & Slap & S0392_1000_13-SRC.pgm & 0.0027 & 0.0033 & 0.0029 & 0.0030 \\
\hline 25 & Slap & S0465_1000_14-SRC.pgm & 0.0029 & 0.0056 & 0.0036 & 0.0043 \\
\hline 26 & Slap & S0466_1000_13-SRC.pgm & 0.0044 & 0.0047 & 0.0045 & 0.0046 \\
\hline 27 & Slap & S0641_1000_14-SRC.pgm & 0.0038 & 0.0060 & 0.0044 & 0.0049 \\
\hline 28 & Slap & S0795_1000_14-SRC.pgm & 0.0096 & 0.0129 & 0.0104 & 0.0113 \\
\hline 29 & Slap & S0817_1000_13-SRC.pgm & 0.0049 & 0.0066 & 0.0053 & 0.0058 \\
\hline 30 & Slap & S0850_1000_14-SRC.pgm & 0.0038 & 0.0054 & 0.0042 & 0.0046 \\
\hline
\end{tabular}




\section{Procedures for Conformance Testing}

Those suppliers wishing to submit SP500-289 implementations of JPEG 2000 CODECs for conformance testing to NIST must follow the procedures outlined in this section. This includes obtaining a NIST assigned encoder identifier (Section 6.1), downloading the NIST reference fingerprint image set (Section 6.2); running the NIST images through the supplier's CODEC and storing the resulting images (Section 6.3); submitting the supplier's CODEC images to NIST for evaluation (Section 6.4).

\subsection{Obtaining a NIST Assigned Encoder Identifier}

Prior to processing certification images, the supplier must obtain a NIST assigned encoder identifier. This identifier must be present in all images encoded by the supplier's CODEC and must be present in conformance test images submitted to NIST for evaluation.

Requests for an encoder identifier must be sent to fingerprintcompression@nist.gov and the request must contain the following information in the subject and body of the message:

Message Subject should be:

JPEG2000 encoder identification request

In the message body please include:

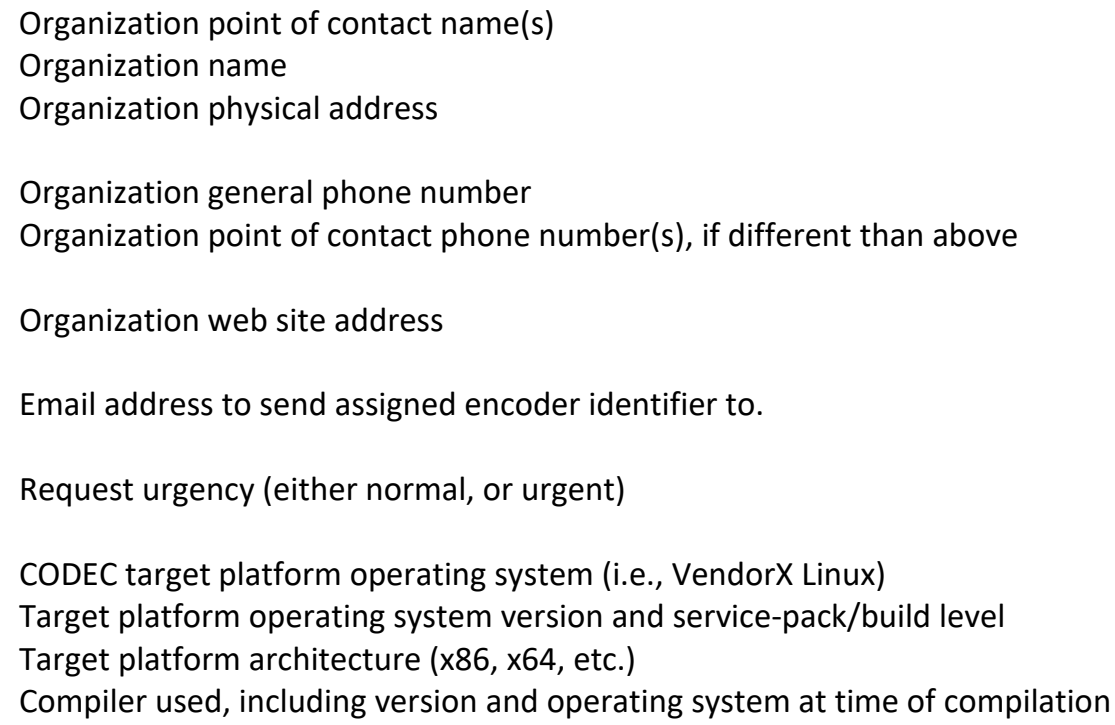

In 7 to 10 days typically, you will electronically receive the 100 byte NIST assigned encoder identifier.

This identifier must be present in all images processed by the supplier's CODEC.

Additionally, the electronic response you will receive from NIST will include a download link for the reference fingerprint image set needed by the supplier for testing. 


\subsection{Downloading NIST Reference Fingerprint Image Set}

The supplier must download a version of the NIST Reference Fingerprint Image Set prepared for use in the conformance test and referred to as the JPEG 2000 Conformance Dataset (J2CD) from NIST. A link to download the NIST J2CD will be provided by NIST in response to the request for NIST assigned vendor identifier (see 6.1).

The reference fingerprint data set will be archived within a portable ZIP format ${ }^{7}$ container and organized as shown in Figure 3. The NIST-provided reference images are stored under the NIST subdirectory. Inside are the thirty source image files $\left(P_{\mathrm{SRC}}\right)$ stored in PGM format. The basenames of these files are what is listed in the fifth column of Table 2, which are appended with a tag and file extension, "-SRC.pgm". There are two additional processed files included with each source file. The first is the corresponding 10:1 lossy compressed file $\left(J_{E_{R}}\right)$ processed using the NIST reference JPEG 2000 CODEC. This file is appended with tag and file extension, "-ER.jp2". The second processed file is the corresponding lossless compressed file using the reference CODEC appended with tag and file extension "-LER.jp2". In all there are 90 reference images included in the download package and listed in Table 22. Initially, the Supplier subdirectory is empty. The processed images generated by the supplier's CODEC will be stored in this folder and the entire package zipped back up and then submitted back to NIST for evaluation.

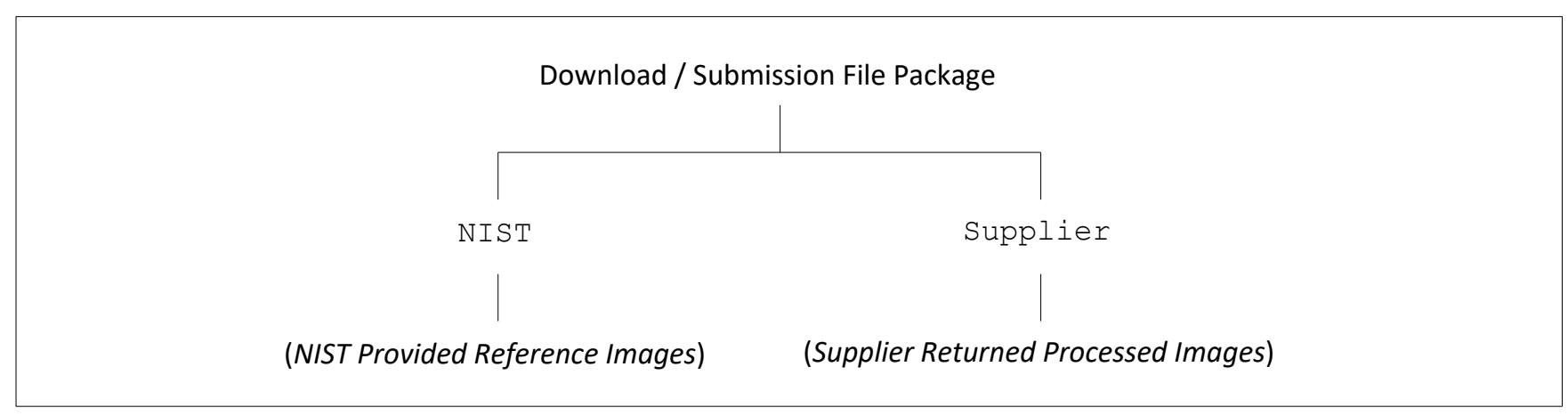

Figure 3 - Download / Submission File Package

\footnotetext{
${ }^{7}$ Per http://www.pkware.com/documents/casestudies/APPNOTE.TXT
} 
Table 22 - NIST JPEG 2000 Conformance Dataset Files

\begin{tabular}{|c|c|c|}
\hline SOURCE FILE & 10:1 PROCESSED FILE $\left(E_{R}\right)$ & LOSSLESS PROCESSED FILE $\left(E_{R}\right)$ \\
\hline S0011_1000_08P-SRC.pgm & S0011_1000_08P-ER.jp2 & S0011_1000_08P-LER.jp2 \\
\hline S0187_1000_07P-SRC.pgm & S0187_1000_07P-ER.jp2 & S0187_1000_07P-LER.jp2 \\
\hline S0201_1000_02P-SRC.pgm & S0201_1000_02P-ER.jp2 & S0201_1000_02P-LER.jp2 \\
\hline S0417_1000_11-SRC.pgm & S0417_1000_11-ER.jp2 & S0417_1000_11-LER.jp2 \\
\hline S0464_1000_09P-SRC.pgm & S0464_1000_09P-ER.jp2 & S0464_1000_09P-LER.jp2 \\
\hline S0564_1000_03P-SRC.pgm & S0564_1000_03P-ER.jp2 & S0564_1000_03P-LER.jp2 \\
\hline S0586_1000_10P-SRC.pgm & S0586_1000_10P-ER.jp2 & S0586_1000_10P-LER.jp2 \\
\hline S0676_1000_12-SRC.pgm & S0676_1000_12-ER.jp2 & S0676_1000_12-LER.jp2 \\
\hline S0704_1000_05P-SRC.pgm & S0704_1000_05P-ER.jp2 & S0704_1000_05P-LER.jp2 \\
\hline S0730_1000_04P-SRC.pgm & S0730_1000_04P-ER.jp2 & S0730_1000_04P-LER.jp2 \\
\hline S0051_1000_05-SRC.pgm & S0051_1000_05-ER.jp2 & S0051_1000_05-LER.jp2 \\
\hline S0093_1000_01-SRC.pgm & S0093_1000_01-ER.jp2 & S0093_1000_01-LER.jp2 \\
\hline S0152_1000_06-SRC.pgm & S0152_1000_06-ER.jp2 & S0152_1000_06-LER.jp2 \\
\hline S0176_1000_02-SRC.pgm & S0176_1000_02-ER.jp2 & S0176_1000_02-LER.jp2 \\
\hline S0249_1000_08-SRC.pgm & S0249_1000_08-ER.jp2 & S0249_1000_08-LER.jp2 \\
\hline S0342_1000_09-SRC.pgm & S0342_1000_09-ER.jp2 & S0342_1000_09-LER.jp2 \\
\hline S0397_1000_04-SRC.pgm & S0397_1000_04-ER.jp2 & S0397_1000_04-LER.jp2 \\
\hline S0503_1000_07-SRC.pgm & S0503_1000_07-ER.jp2 & S0503_1000_07-LER.jp2 \\
\hline S0705_1000_03-SRC.pgm & S0705_1000_03-ER.jp2 & S0705_1000_03-LER.jp2 \\
\hline S0724_1000_10-SRC.pgm & S0724_1000_10-ER.jp2 & S0724_1000_10-LER.jp2 \\
\hline S0139_1000_13-SRC.pgm & S0139_1000_13-ER.jp2 & S0139_1000_13-LER.jp2 \\
\hline S0286_1000_13-SRC.pgm & S0286_1000_13-ER.jp2 & S0286_1000_13-LER.jp2 \\
\hline S0304_1000_14-SRC.pgm & S0304_1000_14-ER.jp2 & S0304_1000_14-LER.jp2 \\
\hline S0392_1000_13-SRC.pgm & S0392_1000_13-ER.jp2 & S0392_1000_13-LER.jp2 \\
\hline S0465_1000_14-SRC.pgm & S0465_1000_14-ER.jp2 & S0465_1000_14-LER.jp2 \\
\hline S0466_1000_13-SRC.pgm & S0466_1000_13-ER.jp2 & S0466_1000_13-LER.jp2 \\
\hline S0641_1000_14-SRC.pgm & S0641_1000_14-ER.jp2 & S0641_1000_14-LER.jp2 \\
\hline S0795_1000_14-SRC.pgm & S0795_1000_14-ER.jp2 & S0795_1000_14-LER.jp2 \\
\hline S0817_1000_13-SRC.pgm & S0817_1000_13-ER.jp2 & S0817_1000_13-LER.jp2 \\
\hline S0850_1000_14-SRC.pgm & S0850_1000_14-ER.jp2 & S0850_1000_14-LER.jp2 \\
\hline
\end{tabular}




\subsection{Running the Testing Protocol}

Upon downloading and unpacking the reference image set, the supplier then executes the testing protocol by running the images systematically through their implementation of the JPEG 2000 CODEC.

\subsubsection{Preparing Files for Encoder Test $1\left(E_{s}\right)$}

Referencing the tests in the order listed in this section, the supplier prepares results for the Encoder Test 1 (Es) by taking each of the source reference files and compressing it using their JPEG 2000 encoder, on one pass using 10:1 lossy compression, and another using lossless compression. The 10:1 lossy compressed image is stored using the source file's basename appended with the tag and extension, "-ES.jp2", while the lossless compressed image is stored using the same basename appended with the tag and extension, "-LES.jp2". Table 23 lists the processed files that are to be generated by this stage and stored under the Supplier subdirectory.

Table 23 - Supplier Prepared Files for Encoder Test 1 (Es)

\begin{tabular}{|l|l|}
\hline 10:1 PROCESSED FILE (Es) & \multicolumn{1}{|c|}{ LOSSLESS PROCESSED FILE (Es) } \\
\hline S0011_1000_08P-ES.jp2 & S0011_1000_08P-LES.jp2 \\
\hline S0187_1000_07P-ES.jp2 & S0187_1000_07P-LES.jp2 \\
\hline S0201_1000_02P-ES.jp2 & S0201_1000_02P-LES.jp2 \\
\hline S0417_1000_11-ES.jp2 & S0417_1000_11-LES.jp2 \\
\hline S0464_1000_09P-ES.jp2 & S0464_1000_09P-LES.jp2 \\
\hline S0564_1000_03P-ES.jp2 & S0564_1000_03P-LES.jp2 \\
\hline S0586_1000_10P-ES.jp2 & S0586_100__10P-LES.jp2 \\
\hline S0676_1000_12-ES.jp2 & S0676_1000_12-LES.jp2 \\
\hline S0704_1000_05P-ES.jp2 & S0704_1000_05P-LES.jp2 \\
\hline S0730_1000_04P-ES.jp2 & S0730_1000_04P-LES.jp2 \\
\hline S0051_1000_05-ES.jp2 & S0051_1000_05-LES.jp2 \\
\hline S0093_1000_01-ES.jp2 & S0093_1000_01-LES.jp2 \\
\hline S0152_1000_06-ES.jp2 & S0152_1000_06-LES.jp2 \\
\hline S0176_1000_02-ES.jp2 & S0176_1000_02-LES.jp2 \\
\hline S0249_1000_08-ES.jp2 & S0249_1000_08-LES.jp2 \\
\hline S0342_1000_09-ES.jp2 & S0342_1000_09-LES.jp2 \\
\hline S0397_1000_04-ES.jp2 & S0397_1000_04-LES.jp2 \\
\hline S0503_1000_07-ES.jp2 & S0503_1000_07-LES.jp2 \\
\hline S0705_1000_03-ES.jp2 & S0705_1000_03-LES.jp2 \\
\hline S0724_1000_10-ES.jp2 & S0724_1000_10-LES.jp2 \\
\hline S0139_1000_13-ES.jp2 & S0139_1000_13-LES.jp2 \\
\hline S0286_1000_13-ES.jp2 & S0286_1000_13-LES.jp2 \\
\hline S0304_1000_14-ES.jp2 & S0304_1000_14-LES.jp2 \\
\hline S0392_1000_13-ES.jp2 & S0392_1000_13-LES.jp2 \\
\hline S0465_1000_14-ES.jp2 & S0465_1000_14-LES.jp2 \\
\hline S0466_1000_13-ES.jp2 & S0466_1000_13-LES.jp2 \\
\hline S0641_1000_14-ES.jp2 & S0641_1000_14-LES.jp2 \\
\hline S0795_1000_14-ES.jp2 & S0795_1000_14-LES.jp2 \\
\hline S0817_1000_13-ES.jp2 & S0817_1000_13-LES.jp2 \\
\hline S0850_1000_14-ES.jp2 & S0850_1000_14-LES.jp2 \\
\hline & \\
\hline
\end{tabular}




\subsubsection{Preparing Files for Decoder Test $2\left(E_{s} D_{s}\right)$}

The supplier prepares results for the Decoder Test $2\left(E_{s} D_{S}\right)$ by taking each of the compressed files from Encoder Test 1 (Table 23) and running them through the supplier's JPEG 2000 decoder. Both the 10:1 lossy compressed and lossless compressed image are decoded and stored as PGM processed files. The decoded 10:1 lossy compressed image is stored using the tag and extension, "-ESDS.pgm", and the decoded lossless compressed image is stored using the tag and extension, "LESDS . pgm". Table 24 lists the processed files that are to be generated by this stage and stored under the Supplier subdirectory.

Table 24 - Supplier Prepared Files for Decoder Test $2\left(E_{s} D_{s}\right)$

\begin{tabular}{|c|c|}
\hline 10:1 PROCESSED FILE $\left(E_{s} D_{s}\right)$ & LOSSLESS PROCESSED FILE $\left(E_{S} D_{S}\right)$ \\
\hline S0011_1000_08P-ESDS.pgm & S0011_1000_08P-LESDS.pgm \\
\hline S0187_1000_07P-ESDS.pgm & S0187_1000_07P-LESDS.pgm \\
\hline S0201_1000_02P-ESDS.pgm & S0201_1000_02P-LESDS.pgm \\
\hline S0417_1000_11-ESDS.pgm & S0417_1000_11-LESDS.pgm \\
\hline S0464_1000_09P-ESDS.pgm & S0464_1000_09P-LESDS.pgm \\
\hline S0564_1000_03P-ESDS.pgm & S0564_1000_03P-LESDS.pgm \\
\hline S0586_1000_10P-ESDS.pgm & S0586_1000_10P-LESDS.pgm \\
\hline S0676_1000_12-ESDS.pgm & S0676_1000_12-LESDS.pgm \\
\hline S0704_1000_05P-ESDS.pgm & S0704_1000_05P-LESDS.pgm \\
\hline S0730_1000_04P-ESDS.pgm & S0730_1000_04P-LESDS.pgm \\
\hline S0051_1000_05-ESDS.pgm & S0051_1000_05-LESDS.pgm \\
\hline S0093_1000_01-ESDS.pgm & S0093_1000_01-LESDS.pgm \\
\hline S0152_1000_06-ESDS.pgm & S0152_1000_06-LESDS.pgm \\
\hline S0176_1000_02-ESDS.pgm & S0176_1000_02-LESDS.pgm \\
\hline S0249_1000_08-ESDS.pgm & S0249_1000_08-LESDS.pgm \\
\hline S0342_1000_09-ESDS.pgm & S0342_1000_09-LESDS.pgm \\
\hline S0397_1000_04-ESDS.pgm & S0397_1000_04-LESDS.pgm \\
\hline S0503_1000_07-ESDS.pgm & S0503_1000_07-LESDS.pgm \\
\hline S0705_1000_03-ESDS.pgm & S0705_1000_03-LESDS.pgm \\
\hline S0724_1000_10-ESDS.pgm & S0724_1000_10-LESDS.pgm \\
\hline S0139_1000_13-ESDS.pgm & S0139_1000_13-LESDS.pgm \\
\hline S0286_1000_13-ESDS.pgm & S0286_1000_13-LESDS.pgm \\
\hline S0304_1000_14-ESDS.pgm & S0304_1000_14-LESDS.pgm \\
\hline S0392_1000_13-ESDS.pgm & S0392_1000_13-LESDS.pgm \\
\hline S0465_1000_14-ESDS.pgm & S0465_1000_14-LESDS.pgm \\
\hline S0466_1000_13-ESDS.pgm & S0466_1000_13-LESDS.pgm \\
\hline S0641_1000_14-ESDS.pgm & S0641_1000_14-LESDS.pgm \\
\hline S0795_1000_14-ESDS.pgm & S0795_1000_14-LESDS.pgm \\
\hline S0817_1000_13-ESDS.pgm & S0817_1000_13-LESDS.pgm \\
\hline S0850_1000_14-ESDS.pgm & S0850_1000_14-LESDS.pgm \\
\hline
\end{tabular}

\subsubsection{Preparing Files for Decoder Test $3\left(E_{S} D_{R}\right)$}

Decoder Test $3\left(E_{S} D_{R}\right)$ will be completed by NIST, using the reference decoder to process each of the files compressed using the Supplier's encoder. As with Decoder Test 2, both the 10:1 lossy compressed and lossless compressed images resulting from Encoder Test 1 will be decoded and evaluated. As the supplier will have already included the files listed in Table 23 under the Supplier subdirectory, no further action is required by the supplier in order to prepare for Decoder Test 3 $\left(E_{S} D_{R}\right)$. 


\subsubsection{Preparing Files for Decoder Test $4\left(E_{R} D_{s}\right)$}

The supplier prepares results for the decoder test $4\left(E_{R} D_{S}\right)$ by taking each of the compressed files generated by the NIST reference encoder and provided within the reference image set (Table 22, columns 2 and 3 ) and running them through the supplier's decoder. Both the 10:1 lossy compressed and lossless compressed image are decoded and stored as PGM processed files. The decoded 10:1 lossy compressed image is stored using the tag and extension, "-ERDS . pgm", and the decoded lossless compressed image is stored using the tag and extension, "-LERDS . pgm". Table 25 lists the processed files that are to be generated by this stage and stored under the Supplier subdirectory.

Table 25 - Supplier Prepared Files for Decoder Test $4\left(E_{R} D_{S}\right)$

\begin{tabular}{|l|l|}
\hline 10:1 PROCESSED FILE (ERDS) & LOSSLESS PROCESSED FILE (ER_DS) \\
\hline S0011_1000_08P-ERDS.pgm & S0011_1000_08P-LERDS.pgm \\
\hline S0187_1000_07P-ERDS.pgm & S0187_1000_07P-LERDS.pgm \\
\hline S0201_1000_02P-ERDS.pgm & S0201_1000_02P-LERDS.pgm \\
\hline S0417_1000_11-ERDS.pgm & S0417_1000_11-LERDS.pgm \\
\hline S0464_1000_09P-ERDS.pgm & S046__1000_09P-LERDS.pgm \\
\hline S0564_1000_03P-ERDS.pgm & S0564_1000_03P-LERDS.pgm \\
\hline S0586_1000_10P-ERDS.pgm & S0586_1000_10P-LERDS.pgm \\
\hline S0676_1000_12-ERDS.pgm & S0676_1000_12-LERDS.pgm \\
\hline S0704_1000_05P-ERDS.pgm & S0704_1000_05P-LERDS.pgm \\
\hline S0730_1000_04P-ERDS.pgm & S0730_1000_04P-LERDS.pgm \\
\hline S0051_1000_05-ERDS.pgm & S0051_1000_05-LERDS.pgm \\
\hline S0093_1000_01-ERDS.pgm & S0093_1000_01-LERDS.pgm \\
\hline S0152_1000_06-ERDS.pgm & S0152_1000_06-LERDS.pgm \\
\hline S0176_1000_02-ERDS.pgm & S0176_1000_02-LERDS.pgm \\
\hline S0249_1000_08-ERDS.pgm & S0249_1000_08-LERDS.pgm \\
\hline S0342_1000_09-ERDS.pgm & S0342_1000_09-LERDS.pgm \\
\hline S0397_1000_04-ERDS.pgm & S0397_1000_04-LERDS.pgm \\
\hline S0503_1000_07-ERDS.pgm & S0503_1000_07-LERDS.pgm \\
\hline S0705_1000_03-ERDS.pgm & S0705_1000_03-LERDS.pgm \\
\hline S0724_1000_10-ERDS.pgm & S0724_1000_10-LERDS.pgm \\
\hline S0139_1000_13-ERDS.pgm & S0139_1000_13-LERDS.pgm \\
\hline S0286_1000_13-ERDS.pgm & S0286_1000_13-LERDS.pgm \\
\hline S0304_1000_14-ERDS.pgm & S0304_1000_14-LERDS.pgm \\
\hline S0392_1000_13-ERDS.pgm & S0392_1000_13-LERDS.pgm \\
\hline S0465_1000_14-ERDS.pgm & S0465_1000_14-LERDS.pgm \\
\hline S0466_1000_13-ERDS.pgm & S0466_1000_13-LERDS.pgm \\
\hline S0641_1000_14-ERDS.pgm & S0641_1000_14-LERDS.pgm \\
\hline S0795_1000_14-ERDS.pgm & S0795_1000_14-LERDS.pgm \\
\hline S0817_1000_13-ERDS.pgm & S0817_1000_13-LERDS.pgm \\
\hline S0850_1000_14-ERDS.pgm & S0850_1000_14-LERDS.pgm \\
\hline
\end{tabular}




\title{
6.4. Submitting Processed Images to NIST for Evaluation
}

Upon supplier's successful completion of the testing protocol, the Supplier subdirectory of the prepared file submission package (Figure 3) must contain all 180 processed files listed in Table 23 through Table 25. The submission package must be zipped and submitted to NIST either electronically or by parcel delivery. The submission package must not contain any executable code, or macro-enabled content. The submission package must not contain any proprietary or sensitive information.

\subsubsection{Submitting Electronically}

The supplier's zipped file package may be submitted to NIST by contacting the official JPEG2000 CODEC Test Custodian fingerprintcompression@nist.gov in order to request an account for the NIST Secure File Transfer Service (or receive alternative instructions). The email's subject line must be: "JPEG2000 encoder conformance submittal"

See https://www.nist.gov/programs-projects/biometric-compression-information for the most up-to-date information on the submission of processed image packages for evaluation.

\subsubsection{Submitting Parcel Post}

The supplier's zipped file package may be submitted to NIST on a data-DVD (DVD-R or DVD+R), and mailed back to NIST using the following address:

\author{
JPEG2000 CODEC Test Custodian \\ ITL-IAD-Image Group \\ 100 Bureau Drive MS 8940 \\ Gaithersburg, MD 20899-8940
}

The supplier's zipped file must not contain any binary executable code and must not include any information deemed proprietary by the supplier. All data submitted to NIST for testing will become the property of NIST.

\subsubsection{FBI Certification}

If the testing protocol is complete and the supplier's results satisfactorily meet the requirements set forth in this document, then NIST notifies the FBI CJIS Division that the supplier received an Overall Result of Pass. Subsequently, the FBI CJIS Division issues a letter certifying that the supplier's implementation of a JPEG 2000 CODEC is conformant with the specification set forth in SP 500-289. An implementation ID and a description of the supplier's submission will then be added to the current list of approved implementations maintained by the FBI.

This implementation ID will remain hard-coded into the supplier solution and included in all JPEG2000 encoded output from the supplier CODEC according to this specification (see section 5.1.3 and section 4.3 of [NIST3] for additional details).

Note: The FBI certification for an implementation will apply only to a specific configuration. A configuration encompasses the software version of the encoder/decoder, hardware platform, operating system, and compiler used. If any of these components change resulting in a binary level change in any of the files the supplier sent to NIST for the purposes of the initial certification, then a recertification including a new implementation ID will be required.

Also note that the certification process is not intended to endorse one implementation over another, but merely to certify that the implementation meets FBI standards. The FBI does not recommend one certified implementation over another. 


\section{References}

\subsection{Publications and Reports}

CHAMBERS Chambers, John; William Cleveland, Beat Kleiner, and Paul Tukey (1983). Graphical Methods for Data

Analysis. Wadsworth

FITZPATRICK Fitzpatrick, M. et al. 1994, "WSQ Compression / Decompression Algorithm Test Report", IAI Annual Conference.

GALTON $\quad$ Galton, F. (2005). Finger prints. Mineola, NY: Dover Publications. (Original work published 1892)

JAIN Jain, A., "Pores and Ridges: High-Resolution Fingerprint Matching Using Level 3 Features", IEEE

Transactions on Pattern Analysis and Machine Intelligence, Vol. 29, No. 1, January 2007.

JASPER JasPer Project JPEG 2000 library. http://www.ece.uvic.ca/ frodo/jasper/

JPEG $\quad$ "T-REC-T.81 : Information technology - Digital compression and coding of continuous-tone still images Requirements and guidelines". http://www.itu.int/rec/T-REC-T.81. Retrieved 2011-01-12.

JPEG2K $\quad$ "ISO/IEC 15444-1:2004 - Information technology -- JPEG 2000 image coding system: Core coding system". http://www.iso.org/iso/iso_catalogue/catalogue_ics/catalogue_detail_ics.htm?csnumber=27687. Retrieved 2009-11-01.

KAKADU Kakadu JPEG 2000 SDK, Kakadu Software, NewSouth Innovations, UNSW Australia, Sydney. http://kakadusoftware.com/

LIBERT “A 1D Spectral Image Validation/Verification Metric for Fingerprints”. Libert, J.M.; Grantham, J.; Orandi, S. August 19, 2009. http://www.nist.gov/customcf/get_pdf.cfm?pub_id=903078. Retrieved 2011-01-12.

LIKERT Likert, R. (1932). A Technique for the Measurement of Attitudes, Archives of Psychology 140, 55.

MTR1 $\quad$ "Profile for 1000 ppi Fingerprint Compression". Lepley, M.A.

http://www.mitre.org/work/tech_papers/tech_papers_04/lepley_fingerprint/lepley_fingerprint.pdf. Retrieved 2011-01-11.

NIST1 $\quad$ "NIST Biometric Image Software". http://Fingerprint.nist.gov/NFIS/. Retrieved 2011-01-12.

NIST2 Orandi, S., Libert, J. M., Grantham, J. D., Ko, K., Wood, S.S., Wu, J. Effects of JPEG 2000 Image Compression on 1000 ppi Fingerprint Imagery, NIST Interagency Report 7778, National Institutes of Standards and Technology, Gaithersburg, MD. http://www.nist.gov/customcf/get pdf.cfm?pub id=911122. Retrieved on 09/01/2012.

NIST3 Orandi, S., Libert, J., Grantham, J., Ko, K., Wood, S., Byers, F., Bandini, B., Harvey, S., Garris, M. Compression Guidance for 1000 ppi Friction Ridge Imagery, NIST Special Publication 500-289, National Institutes of Standards and Technology, Gaithersburg, MD. http://nvlpubs.nist.gov/nistpubs/specialpublications/NIST.SP.500-289.pdf . Retrieved on 09/15/2014

NIST6 G. Fiumara, P. Flanagan, J. Grantham, B. Bandini, K. Ko, and J. Libert, NIST Special Database 300: Uncompressed Plain and Rolled Images from Fingerprint Cards, NIST Technical Note 1993, 2018.

OPENJPEG "OpenJPEG library : an open source JPEG 2000 codec". http://www.openjpeg.org/index.php?menu=news. Retrieved 2011-01-12.

PGM “PGM Format Specification”. http://netpbm.sourceforge.net/doc/pgm.html. Retrieved 2015-01-23

SHAPIRO Shapiro, S. S.; Wilk, M. B. (1965). "An analysis of variance test for normality (complete samples)". Biometrika52 (3-4): 591-611. doi:10.1093/biomet/52.3-4.591. JSTOR 2333709MR205384.

WSQ1 $\quad$ "WSQ Gray-Scale Fingerprint Image Compression Specification" Version 3.1.

https://www.fbibiospecs.org/docs/WSQ_Gray-scale_Specification_Version_3_1.pdf. Retrieved 2010-0111. [TODO: Need reference to 289]

WSQ2 WSQ Fingerprint Image Compression Encoder/Decoder Compliance Guidelines, NIST Web Site Reference for WSQ Compliance Testing; http://www.nist.gov/itl/iad/ig/wsq.cfm . Retrieved 2015-04-06. 


\section{Appendix A. PGM Profile}

Portable Graymap Format (PGM) is one of several image formats defined by Netpbm [PGM], an open-source programming library. PGM is a widely supported and very straight-forward image format consisting of a small, simplified header followed by uncompressed 8- or 16-bit raw image data in ASCII or binary encodings.

A valid PGM file header always begins with a two-byte string which indicates that the file is a PGM image containing grayscale image data encoded in either ASCII ("P2") or binary ("P5"). Binary-encoded files are generally smaller in size and are preferred in most cases, including the storage of fingerprint images. The two-byte format identification string is followed by decimal values defining the width, height, and maximum gray value; each separated by whitespace characters such as spaces, tabs, carriage-returns (CRs) or line-feeds (LFs). The header is always encoded as ASCII and is terminated by a whitespace character, which also marks the beginning of the image data (which may be either ASCII- or binary-encoded). The header may also contain comments as strings beginning with the '\#' character and terminated by a CR or LF character.

Table 26 - PGM File Structure and Metadata

\begin{tabular}{|l|l|l|l|}
\hline PGM Data Field & Value & Size (Bytes) & Hexadecimal \\
\hline PGM Signature & 'P5' & 3 & $50350 \mathrm{~A}$ \\
\hline Comment & Comment String & variable & \\
\hline Width & Image Width (pixels) & variable & \\
\hline Height & Image Height (pixels) & variable & \\
\hline Max Value & $' 255^{\prime}$ & 4 & $3235350 \mathrm{~A}$ \\
\hline
\end{tabular}

